\title{
Interaction of nanoparticles with lipid films: the role of symmetry
}

Lucrezia Caselli ${ }^{a}$, Andrea Ridolfi ${ }^{a . b}$, Gaetano Mangiapia $^{c}$, Pierfancesco Maltoni ${ }^{\text {at }}$, Jean-Francois Moulin ${ }^{c}$, Debora Berti ${ }^{a}$, Nina-Juliane Steinke ${ }^{d}$, Emil Gustafsson ${ }^{e}$, Tommy Nylander ${ }^{f *}$ and Costanza Montis ${ }^{\text {a* }}$

${ }^{a}$ Department of Chemistry, University of Florence and CSGI, Florence (Italy)

b ISMN-CNR and CSGI, Bologna (Italy)

c German Engineering Materials Science Centre (GEMS) at Heinz Maier-Leibnitz Zentrum (MLZ), Helmholtz-Zentrum Hereon, Lichtenbergstr. 1, 85748 Garching bei München, Germany

${ }^{a}$ ISIS, Rutherford Appleton Laboratory Didcot, Oxfordshire (UK)

e Department of Chemistry, Uppsala University, Uppsala (Sweden)

${ }^{\dagger}$ Department of Chemistry, Lund University, Lund (Sweden)

† Current affiliations: P. M. Department of Materials Science and Engineering, Uppsala University, Uppsala (Sweden); N.-J. S. Institut Laue-Langevin, Grenoble (France)

When nanomaterials are put in contact with living organisms, their interaction with biomacromolacules and biological barriers will determine their bioactivity, biological fate and cytotoxicity. In this context, the role of symmetry/shape anisotropy of both the nanomaterials and the biological interfaces on their interaction mechanism, is a relatively unaddressed issue. Here, we study the interaction of gold nanoparticles (NPs) of different shape (nanospheres and nanorods) with biomimetic membranes of different symmetry, i.e. flat membranes (of $2 \mathrm{D}$ symmetry, representative of the most common plasma membrane geometry), and cubic membranes (of 3D symmetry, representative of non-lamellar membranes, encountered in Nature in peculiar biological conditions). Through an ensemble of structural techniques (Neutron Reflectometry, Grazing Incidence Small-Angle Neutron Scattering), we found that, on a nanometric lengthscale, the structural stability of the membrane towards NPs is dependent on the topological characteristic of the lipid assembly and of the NPs, with higher symmetry related to higher stability. In addition, Confocal Laser Scanning Microscopy analyses highlight, on a micrometric legthscale, that cubic and lamellar phases interact with NPs according to two distinct mechanisms, related to the different structures of the lipid assemblies. This study represents a 
first attempt to systematically study the role of membrane symmetry on the interaction with NPs; the results will contribute to improve the fundamental knowledge on nano-bio interfaces and, more in general, will provide new insights on the biological function of non-lamellar cubic arrangements of interfacial membranes as a response strategy.

\section{Introduction}

Nanomaterials, either intentionally administered or unintentionally released in the environment, are increasingly exposed to living organisms. The interaction of nanosystems with biological matter proceeds through an intricate balance of forces ${ }^{1-3}$, occurring at the nano-bio interface, where nanomaterials, e.g. nanoparticles, meet biological fluids and barriers (such as biological membranes). Disentangling these contributions is key to understand the fate of the nanoparticles in living systems, in particular their toxic effects and, concerning nanomaterials designed for nanomedicine, their efficacy in reaching their biological target ${ }^{4-10}$. In this context, where multiple factors and forces are contemporary at play, the concept of symmetry, which can be related both to the shape of NPs and to the nanoscale structure of the target biological membrane, is particularly interesting.

NPs shape' asymmetry is currently the focus of a fervent research: both experimental and computational studies highlight that the anisotropy of NPs is a determining factor in their internalization pathways in cells ${ }^{4,11-15}$. In particular, NPs shape modifies the area available for NPs adsorption on lipid surfaces ${ }^{16}$, modulating the strength of NP-membrane adhesion forces. Additionally, topological effects are significant in the surface functionalization of NPs (e.g., ligand surface density), affecting their chemical identity ${ }^{17}$. Finally, a high surface curvature, either uniformly distributed on the surface of NPs (e.g., small NPs with sizes of a few nm) or localized at the sharp edges of asymmetric NPs (e.g., nanorods), is associated to higher energetic costs in terms of wrapping and internalization by membranes ${ }^{3,12,14,15}$ : anisotropic NPs, unlike spheres, can reorient in proximity to the lipid interface to minimize this energy penalty and maximize the adhesion strength to the membrane. ${ }^{18}$

On the other side, biological membranes are commonly characterized by a planar geometry with a bidimensional structure (an infinite plan constituted by a lipid bilayer); however, such arrangement can undergo striking temporary or permanent topological modifications in selected conditions (for instance in cell trafficking phenomena or in pathological conditions) which are often related to a significant alteration in the symmetry of the lipid self-assembled construct. In 
particular, curved membrane configurations characterized by a 3D symmetry, as cubic bicontinuous arrangements, are known to occur in cells under pathological conditions (e.g., drug detoxification, starving, infection, oxidative stress, and cancer disease) or during certain phases of cell life (e.g. membrane fusion) ${ }^{19-21}$. Up to now, the investigations related to these so-called "cubic membranes" have been limited to a descriptive level, while their biological function remains unexplored ${ }^{20,21}$. This is mainly due to the transient nature of non-lamellar biological membranes, which makes their investigation in natural systems very challenging ${ }^{21}$. In this framework, lipid models of synthetic nature, mimicking cubic membranes' structure, can be used to simplify the investigation. Our recent findings ${ }^{22-24}$ show that it is possible to obtain solid-supported lipid model surface layers of cubic symmetry, with controlled physicochemical and structural features, enabling the study of NPs-cubic membranes in highly simplified and controlled conditions.

In this contribution, we investigate how gold nanoparticles (AuNPs) of different symmetry (namely gold nanospheres (AuNSs) and gold nanorods (AuNRs)) but similar size and surface coating, interact with target lipid films of different internal structure (namely a lamellar phase, of 2D symmetry, and a cubic phase, of 3D symmetry). By combining neutron scattering techniques (Neutron Reflectivity (NR) and Grazing-Incidence Small-Angle Neutron Scattering (GISANS)) with Confocal Laser Scanning Microscopy (CSLM), we investigate NPs-lipid films interaction at different interaction times (from few minutes to many hours) and at different lengthscales (from nanometers to micrometers).

Our results show that the shape and symmetry of both the NPs and the lipid mesophase are key factors in driving the mechanism and strength of NP-lipid film interaction. Overall, this finding will contribute at improving our comprehension of the phenomena occurring at the nano-bio interface. More in general, it will provide insights into the role of cubic membranes in biological systems.

\section{Results and discussion}

\section{Characterization of gold nanoparticles and lipid films of different symmetry}

Cationic gold nanospheres (AuNSs) and nanorods (AuNRs) in water were prepared according to well-known synthetic routes ${ }^{25-28}$. The concentration of particles was determined by ICP-AES, as described in the SI. Figure 1, Panel A shows Small Angle X-ray Scattering (SAXS) profiles and Transmission Electron Microscopy (TEM) images of both AuNSs and AuNRs. The SAXS data for the AuNSs were analysed with a Schultz spheres form factor and they were found to be 
polydisperse spheres (PD 0.4) with a metal core of $3.4 \mathrm{~nm}$, in perfect agreement with TEM $(d=$ $3.4 \pm 0.6 \mathrm{~nm}$ ). AuNRs SAXS data were analysed by applying a model with the form factor for cylinders with a polydisperse cross section. The obtained fitting parameters are consistent with 7.4 (length/width) aspect ratio nanorods (length $19.2 \mathrm{~nm}$, diameter $2.6 \mathrm{~nm}$ (PD 0.2)), in line with TEM analysis (length $18 \pm 5 \mathrm{~nm}$, diameter $4 \pm 1 \mathrm{~nm}$ ). The cationic capping agents of AuNSs and AuNRs (mearcaptoundecyl-N,N,N-trimethyl ammonium bromide (TMA) and Cetyltrimethyl ammonium bromide (CTAB), respectively) determine for both spheres and rods an overall positive zeta potential (+ $26 \pm 1 \mathrm{mV}$ for AuNSs, $+45 \pm 2 \mathrm{mV}$ for AuNRs). In summary, both AuNSs and AuNRs are characterized by a net positive charge, which has been shown to result in a strong attractive interaction with zwitterionic phospholipid membranes, characterized by a slightly negative zeta potential ${ }^{1,29}$. In addition, spherical NPs are characterized by a diameter similar to the rods cross section; therefore, the main difference between AuNSs and AuNRs is the strong asymmetry of AuNRs, with two curved surfaces (the poles), separated by a cylindrical body.
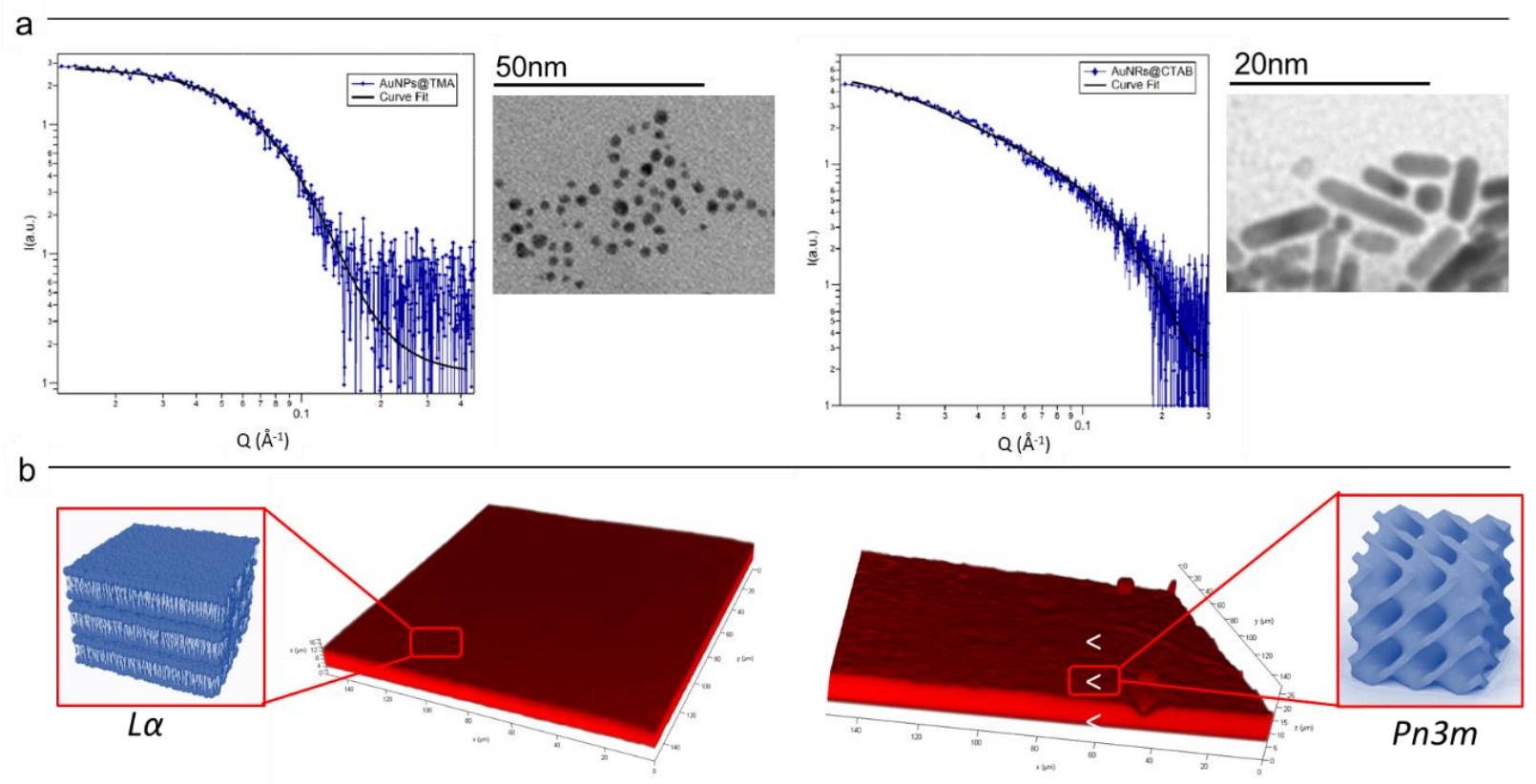

Figure 1 Panel A) Physicochemical properties of NPs. Left: SAXS profile of AuNSs in water (at a concentration of $1.1 \cdot 10^{15}$ particles $/ \mathrm{ml}$ ) and the corresponding fit of the Schultz polydisperse spheres model to the experimental data (see SI for details). The inset shows a typical TEM image of AuNSs; Right: SAXS profile of AuNRs in water (at a concentration of $2.4 \cdot 10^{14}$ particles $/ \mathrm{mL}$ ) and curve fitting according to a Cylinder poly radius model. The inset reports a TEM image of AuNRs; Panel B) Physicochemical properties of lipid films. Three-dimensional reconstruction of a confocal fluorescence z-stack of images of the lamellar (left side) and the cubic (right side) films (tilted surface area of $150 \times 150$ $\mu \mathrm{m})$. The insets depict the lamellar La (left side) and cubic Pn3m (right side) structures. 
Lipid films on glass coverslips of different liquid crystalline structure were prepared by spin-coating n-hexane solutions of 1-monoolein (GMO) to produce the cubic phase films and of GMO/DOPC (1,2-Dioleoyl-sn-glycero-3-phosphocholine) (50/50 mol \%) for lamellar phase films. For CLSM experiments, a fluorescent hydrophobic dye (Nile Red, $0.1 \mathrm{~mol} \%$ with respect to total lipid amount) was added. The lipid films were then dried in vacuum to completely remove the solvents and subsequently hydrated with excess water. Figure 1 , Panel B, displays the 3D reconstructions of fully hydrated GMO and GMO/DOPC films.

The morphology of the films at the micron-scale appears very similar, i.e., a homogeneous thickness (of $10 \pm 2 \mathrm{~mm}$ for both films), with low roughness. Due to the different composition, it is expected that the lipid films are characterized by a different structural arrangement at a nanometric length scale: a Pn3m cubic phase for GMO films and a La lamellar phase for GMO/DOPC films (see the schemes in Figure 1B), which was confirmed by neutron reflectometry as described in the following section.

\section{Nanoparticles-lipid films interaction at a nanometric lengthscale}
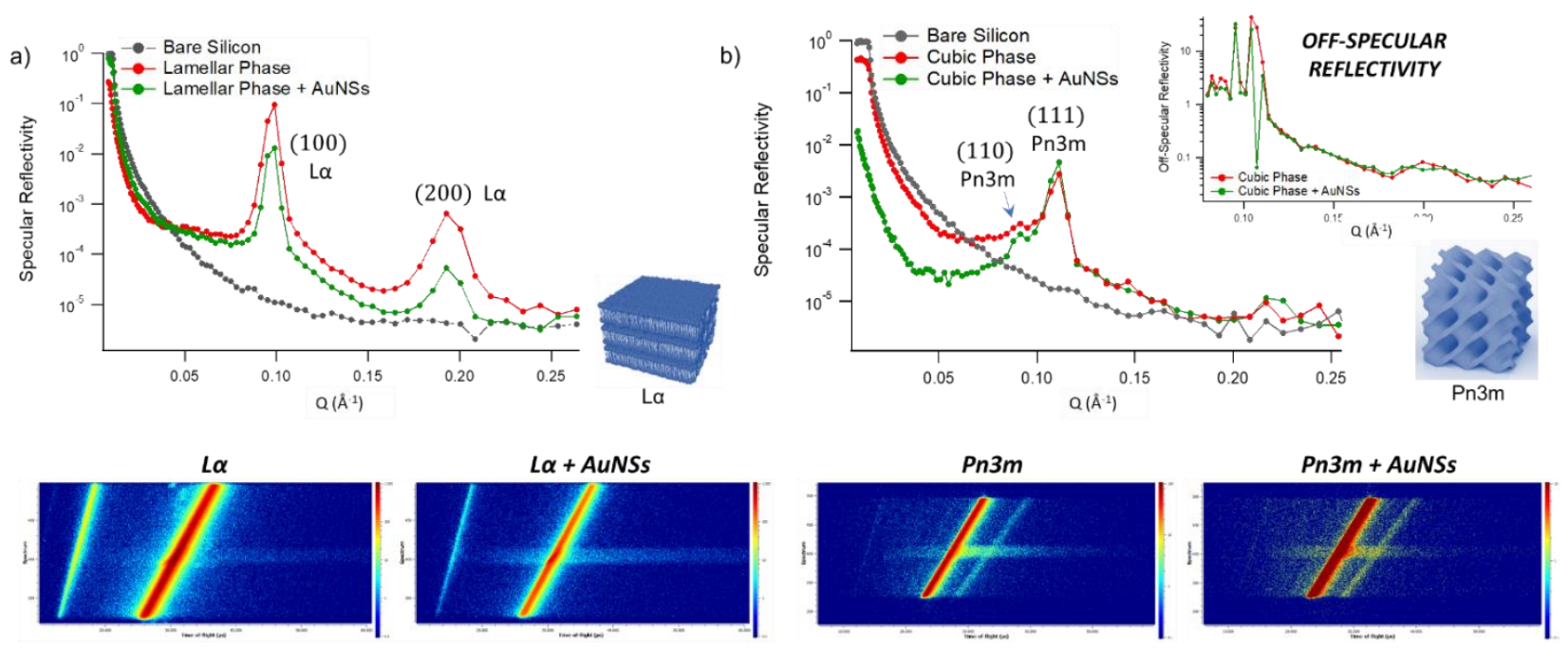

Figure 2 Panel A) Structural characterization of GMO/DOPC films interacting with AuNSs. Top: Specular reflectivity profiles of the GMO/DOPC film in the absence and in the presence of AuNSs, together with reflectivity of the bare silica surface. The inset sketches the inner La structure of the film; Bottom: images of the reflectivity detectors acquired for the La phase in the absence and in the presence of AuNSs; Panel B) Structural characterization of GMO films interacting with AuNSs. Specular reflectivity of the GMO film in the absence and in the presence of AuNSs, together with reflectivity of the bare silica surface. The top inset represents the Off-specular reflectivity of GMO films in the absence and in the presence of AuNSs, while the bottom inset sketches the inner cubic Pn3m structure of the lipid film; Bottom: images of the reflectivity detector acquired for the Pn3m phase in the absence and in the presence of AuNSs. Measurements acquired at OFFSPEC 30, ISIS Neutron and Moun Source (United Kingdom). 
GMO and GMO/DOPC films were formed on ultra-polished silicon blocks and fully hydrated with excess $\mathrm{D}_{2} \mathrm{O}$ by equilibrating for 12 hours before recording characterisation with NR. Subsequently, AuNSs in $\mathrm{D}_{2} \mathrm{O}$ were pumped in the measurement cell at a concentration of $3 \cdot 10^{14}$ particles $/ \mathrm{mL}$ (corresponding to $0.12 \mathrm{mg} / \mathrm{mL}$ gold concentration). After 8 hours incubation, the reflectivity curve for the lipid films in the presence of AuNSs was recorded.

The reflectivity profiles of GMO/DOPC (Figure 2a) and GMO (Figure 2b) films without NPs are consistent with the formation of highly ordered mesophases, with multiple Bragg reflections. In particular, the reflectivity profile of the GMO-DOPC film features two prominent Bragg peaks located at 0.099 and $0.192 \AA^{-1}$, corresponding to the first two reflexes of the lamellar La phase. As sketched in Figure 2a (left inset), the lamellar structure of the micrometric film consists of a stack of flat lipid bilayers separated by water layers. The unit cell spacing was calculated using the $Q$-position of the maximum intensity first-order reflexes ${ }^{30}$ (see $\mathrm{SI}$ for details), yielding a value of $64 \pm 1 \AA$. This value is consistent with previous results obtained for a bulk GMO/DOPC Laphase ${ }^{31}$. Considering a thickness of about 34-37 $\AA$ for the GMO/DOPC bilayer (based on $37 \AA$ and $34 \AA$ for DOPC and GMO bilayers ${ }^{32,33}$, respectively), the water interlayers will have a thickness of 26-29 A. The reflectivity profile of the GMO lipid film (Figure $2 b$ ) presents at least two clearly distinguishable Bragg peaks, indicating also in this case a highly ordered internal structure. The position of these two peaks are at $Q$-values 0.091 and $0.111 \AA^{-1}$. These $Q$ values can be associated to an inverse cubic phase with crystallographic space group Pn3m, corresponding to Miller indices (110) and (111). As depicted in the right inset, this structure has a bicontinuous nature, featuring a single lipid bilayer with negative interfacial curvature, which divides the inner space into two sets of interwoven aqueous channels. The lattice spacing $d$, calculated as described in the SI is $95 \pm 0.5 \AA$, corresponding to a water channel radius of $22 \AA 34$.

When AuNSs are added to the GMO/DOPC film, the reflectivity still shows Bragg reflection peaks typical of the lamellar arrangement. Although the positions are unchanged compared to before adding the particles, the presence of AuNSs produces a significant reduction in the peaks' intensity. This suggests that a partial disruption of the bilayer arrangement occurs (a possible effect of lipid removal when the liquid flow through the sample cell was previously ruled out with a control experiment). However, the same AuNSs seem to have negligible impact on the nanostructure of the cubic lipid film: while a modification of the curve at low $Q$ might suggest a variation of the overall film thickness and roughness, the presence of AuNSs does not significantly modify neither the position nor the intensity of the Bragg peaks, compared to the neat GMO film. This suggests that the nanostructure in the cubic film is stable and does not change even after 13 
hours of interaction ( $8 \mathrm{~h}$ of incubation $+5 \mathrm{~h}$ of measurement) with the AuNSs. We further investigated the structure of AuNSs/cubic phase films by means of GISANS. The aim was to reveal possible effects of AuNSs on cubic phases, occurring over longer time (8 h of incubation + $24 \mathrm{~h}$ of measurement, for a total AuNSs-membrane interaction time of $32 \mathrm{~h}$ ). This technique allows also investigating the in-plane lipid arrangement and how it changes on the AuNSs injection ${ }^{35}$. Lamellar films only have structural order with respect to the normal of the layers, while cubic phases are also characterized by high lateral ordering, whose possible modifications can be easily detected by GISANS. We performed GISANS on a neat GMO cubic film in $\mathrm{D}_{2} \mathrm{O}$, previously equilibrated for $12 \mathrm{~h}$ (Figure 3a). Then, we added AuNSs at the same concentration as used for the NR measurements and left to incubate for $8 \mathrm{~h}$.
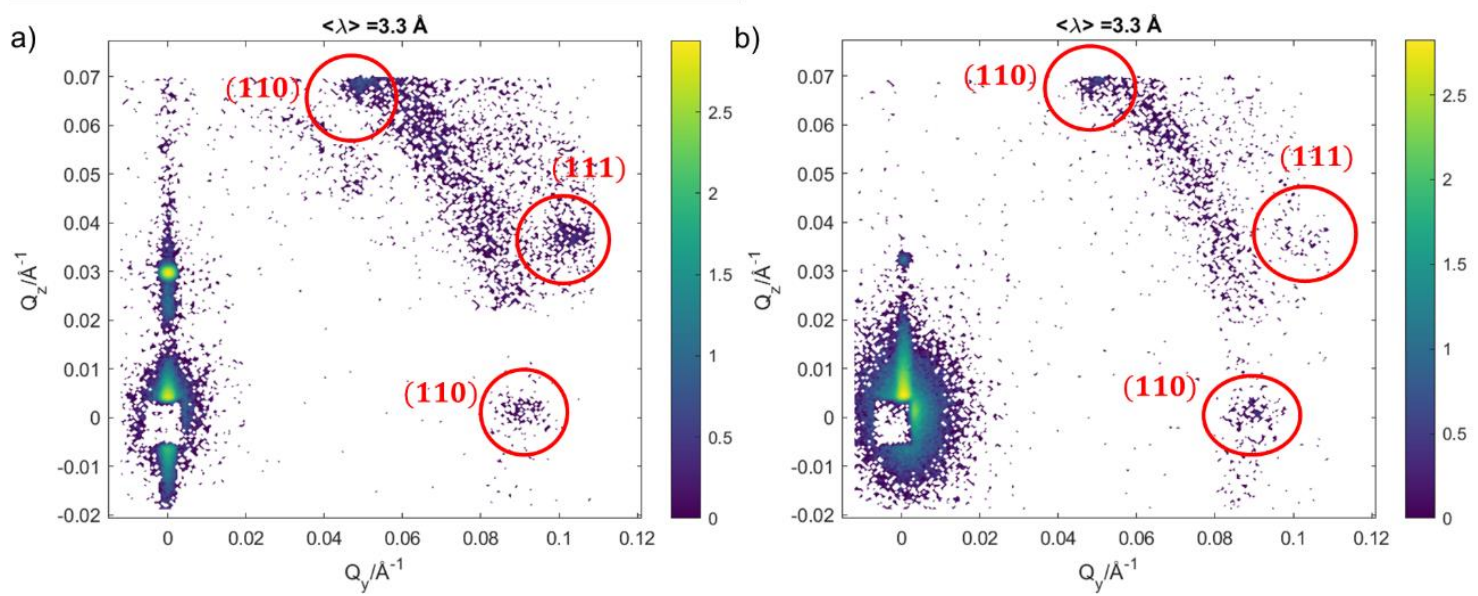

Figure 3 a) GISANS pattern of GMO cubic Pn3m films, in the absence of NPs; b) GISANS pattern of the GMO cubic Pn3m phase in the presence of AuNSs. Measurements acquired at REFSANS, Heinz Maier-Leibnitz Zentrum, Garching, Germany. In both the patterns, intensities are reported in logarithmic scale.

The $Q_{z} / Q_{y}$ GISANS pattern of the GMO film in the absence of AuNSs shows isolated spots, which represent the (110) and (111) Bragg reflections of the Pn3m structure. By performing horizontal line cuts (i.e. along $Q z$ ) from the 2D GISANS plot, we determined the position of these highest intensity points (see Figure S1 of SI for details), whose corresponding $Q$ values describe a Pn3m arrangement with a lattice parameter of $102 \AA$, in good agreement with the NR analysis. The presence of a spot-like pattern suggests a oriented cubic structure at the interface ${ }^{36}$, rather than a typical powder pattern that is mostly the case for the corresponding bulk GMO. The addition of AuNSs (Figure 3b) smears out the spot-like pattern, which is now barely distinguishable from the Debye-Scherrer ring. This suggests a significant increase of the structural disorder within the mesophase, which can be either due to a partial disruption of the cubic symmetry or to a loss of spatial orientation, as well as loss of material from the surface. Interestingly, we also highlighted 
a significant shrinkage of the cubic phase's lattice parameter in the presence of AuNSs (i.e., 2 $\mathrm{nm}$ ) (see Figure S2 of SI), implying a dehydration of the structure induced by AuNSs. Therefore, GISANS data reveals a non-negligible impact of AuNSs on the cubic structure, when observed on longer time scales, thus complementing NR data. In summary, for (relatively) short interaction times (NR data), the structural order of the lamellar mesophase is decreased, while, in the same timeframe, the cubic phase mesostructure appears preserved. However, for longer incubation times (GISANS), AuNSs also affect the structural order of the cubic film. Therefore, the cubic mesophase appears significantly more stable than the lamellar one, when subjected to AuNSs exposure.
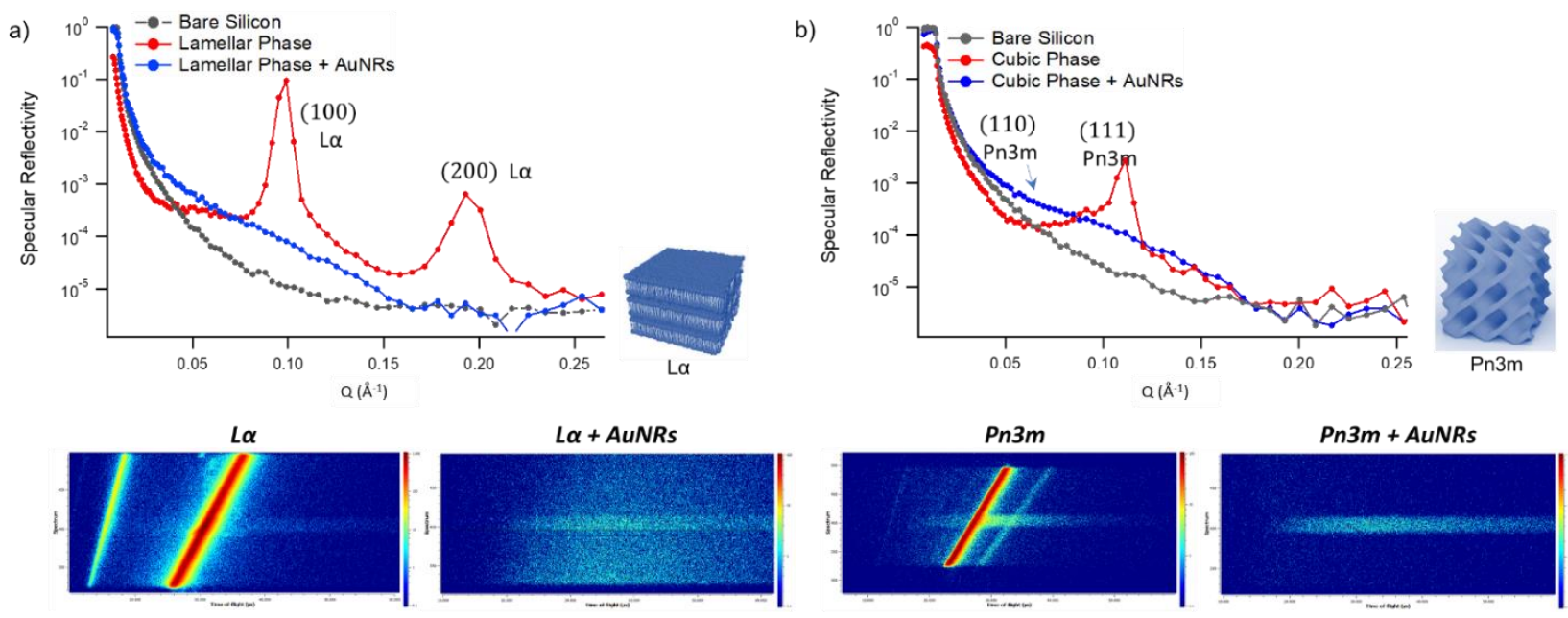

Figure 4 Panel A) Structural characterization of GMO/DOPC films interacting with AuNRs. Top: Specular reflectivity profiles of the GMO/DOPC film in the absence (red curve) and in the presence of AuNRs, together with reflectivity of the bare silica surface. The inset sketches the inner La structure of the film; Bottom: Detector images acquired for the La phase in the absence and in the presence of AuNRs; Panel B) Structural characterization of GMO films interacting with AuNRs. Specular reflectivity of the GMO film in the absence and in the presence of AuNRs, together with reflectivity of the bare silica surface. The inset sketches the inner cubic Pn3m structure of the lipid film; Bottom: Detector images acquired for the Pn3m phase in the absence and in the presence of AuNRs. Measurements acquired at OFFSPEC 30, ISIS Neutron and Moun Source (United Kingdom).

Figure 4 shows NR data obtained for the same lipid films (lamellar in Figure 4a and cubic in Figure 4b) exposed to AuNRs at a concentration of particles/ $\mathrm{mL}$ (corresponding to a Au concentration of $0.12 \mathrm{mg} / \mathrm{mL}$ ), measured in the same experimental conditions as AuNSs. In contrast to AuNSs, AuNRs completely destroy both the lamellar and cubic arrangements, as evident from the absence of Bragg reflexes in the corresponding reflectivity profiles: indeed, in both lamellar film and cubic film the NR profile of the remaining layer is ascribable to the presence of a single bilayer of thickness consistent with a pure GMO or GMO/DOPC bilayer (curve fitting results are in the SI, Figure S2). AuNRs and AuNSs are characterized by similar size (similar cross section of AuNRs 
and AuNSs diameter) and by a net cationic surface charge. Therefore, this enhanced interaction effect of AuNRs with lipid films suggests a strong role played by NPs shape asymmetry in the interaction with model lipid films.

Clearly, the drastic effect of AuNRs is not dependent on whether the film has a lamellar or a cubic phase structure, in the time frame of our experimental observations. To gain further insight on this fast disruption process, we performed Neutron Reflectivity kinetics studies, allowing for monitoring the structural alteration produced by AuNRs on shorter time scales ${ }^{37}$. We measured the reflectivity of lipid films in $\mathrm{H}_{2} \mathrm{O}$, just before and after the injection of AuNRs within the measuring cell, following the change in reflectivity at time intervals of 2.5 minutes, covering a time period over of 5 hours. Representative data are shown in Figure 5 as the $(Q z, Q x)$ images from the reflectivity measurements for the lamellar (Panel a) and cubic film (Panel b), before and AuNR addition.

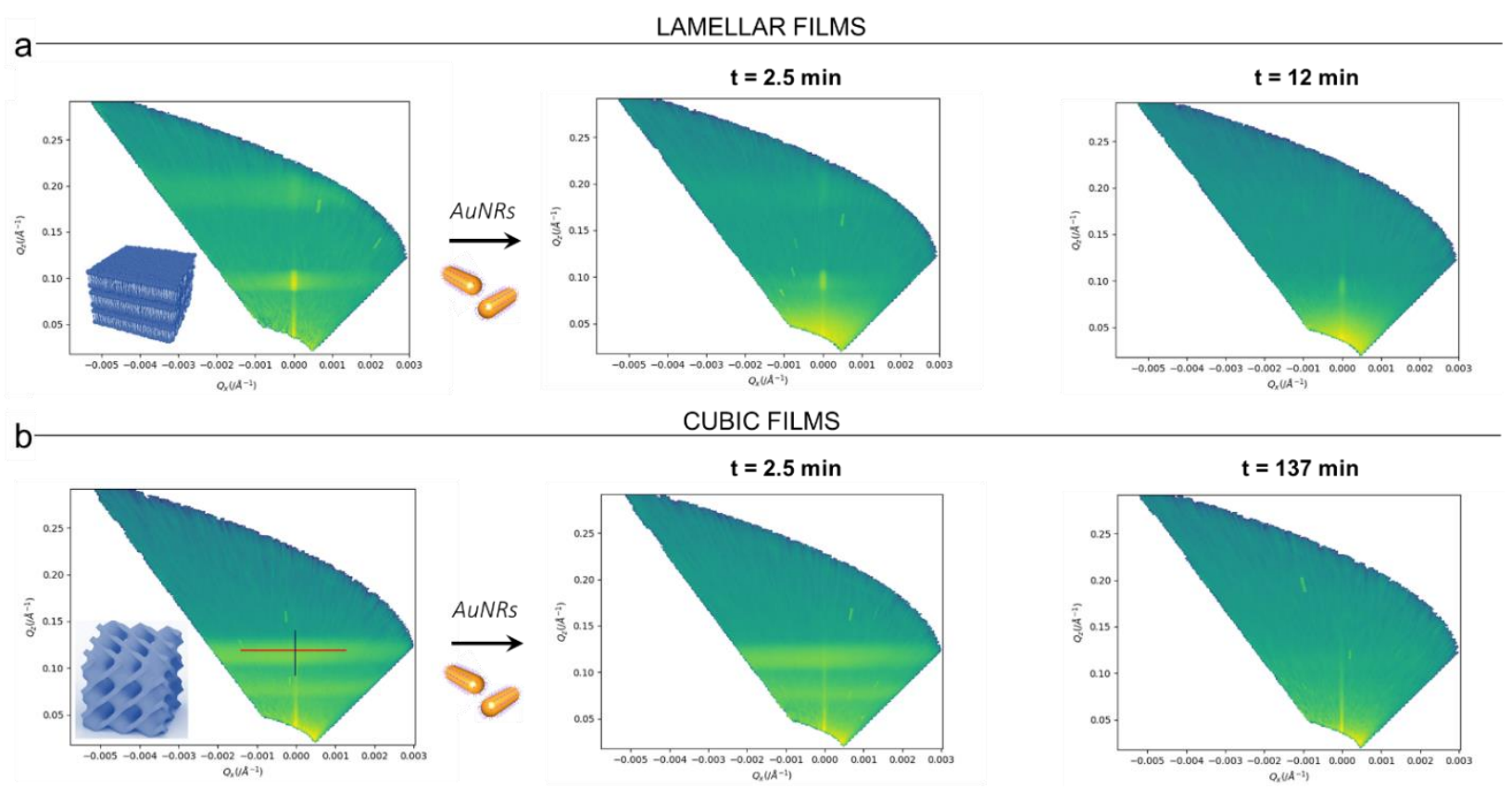

Figure 5 Panel A) Structural characterization of GMO/DOPC films interacting with AuNRs at short time-frames: $(Q z, Q x)$ representations of the off-specular scattering of a lamellar film in the absence of AuNRs and at different times from the injection of AuNRs (acquisition in $\mathrm{H}_{2} \mathrm{O}$ ). Panel B) Structural characterization of GMO films interacting with AuNRs at short time-frames: $(Q z, Q x)$ representations enhancing the off-specular scattering of a cubic film in the absence of AuNRs and at different times from the injection of AuNRs (acquisition in $\mathrm{H}_{2} \mathrm{O}$ ). The integrated intensity along the specular reflectivity line (see blue line in the left $\left(Q_{z}, Q_{x}\right)$ plot of Panel $B$ ) gives the specular reflectivity profile of lamellar and cubic films.

The $(Q z, Q x)$ maps of both lamellar and cubic films in the absence of NPs show pronounced offspecular patterns, featuring well defined "Bragg sheets" (see the red line in the left $(Q z, Q x)$ plot of Panel B, as an example). The high intensity of the off-specular Bragg sheets allowed us to monitor the impact of AuNRs on the structure of lipid films over time. Panels $a$ and $b$ highlight $a$ strong effect of AuNRs on both lamellar and cubic arrangements. The main effect is smearing out 
of the characteristic Bragg sheets with time that ultimately leads to the complete loss of the offspecular signal. This indicates total disruption of the films internal structure. Interestingly, lamellar films completely lose their structural organization significantly faster than cubic ones, with Braggs sheets completely vanishing within $12 \mathrm{~min}$. On the contrary, cubic phase off-specular signal is still detectable after more than $2 \mathrm{~h}$ of incubation with AuNRs. Thus, cubic phases show larger structural stability when exposed to rods lamellar ones. This is consistent with the previous reported effect of spherical particles.

In summary, NR and GISANS data allowed characterizing NPs-lipid films interactions at a nanoscale level, revealing that: (i) AuNRs are more effective than AuNSs in inducing structural modifications in the lipid films (ii) cubic phases are more stable than lamellar phases in this respect. The first point (i) is consistent with other studies reported in literature. Indeed, several computational and experimental studies highlighted that AuNRs exhibit a stronger interaction with lipid membranes and that this a consequence of the anisotropic shape of NPs ${ }^{38,39}$. In addition, our results demonstrate that this behaviour of AuNRs does not depend the lipid assembly phase, i.e., it applies both to planar membranes and to bilayers which are highly curved. On the other hand, the higher stability of cubic phases towards the interaction with NPs might be counterintuitive. In fact, cubic phase- nanoparticle dispersions (cubosomes) are known to be particularly prone to attach to target membranes, where lipid exchange can occur. ${ }^{40}$ For the case with AuNP, the adhesion of NPs to an already curved membrane might be more favourable if the curvature of the particle matches that of the lipid aqueous interface. We further investigated this aspect on the micron-scale by studying the same systems with Confocal Laser Scanning Microscopy (CLSM)- This allowed us to follow the morphological modification induced by NPs on lipid films.

\section{Nanoparticles-lipid films interaction at a micrometric length scale}

We performed the CLSM study over the same time frame of Neutron kinetics measurements. To this purpose, fluorescently labelled lamellar and cubic phases (Figure 1, panel B) were imaged before and after the injection of NPs. Figure 6 gathers representative lateral-view CLSM images of a lamellar film exposed to AuNSs (Figure 6a) and AuNRs (Figure 6b). The action of spherical AuNSs (added at the same concentration employed in NR experiments) produces an initial swelling of the film (20 min), which increases the distance between the different lamellae composing the structure (Figure 6a). 


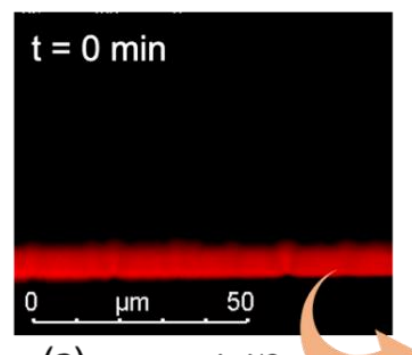

(a)

AunSs

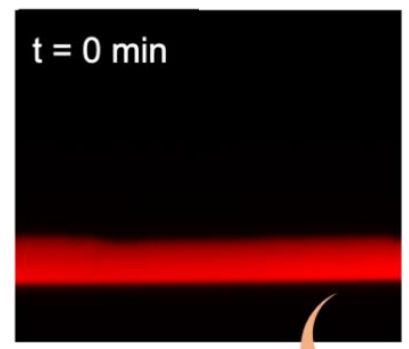

(b)
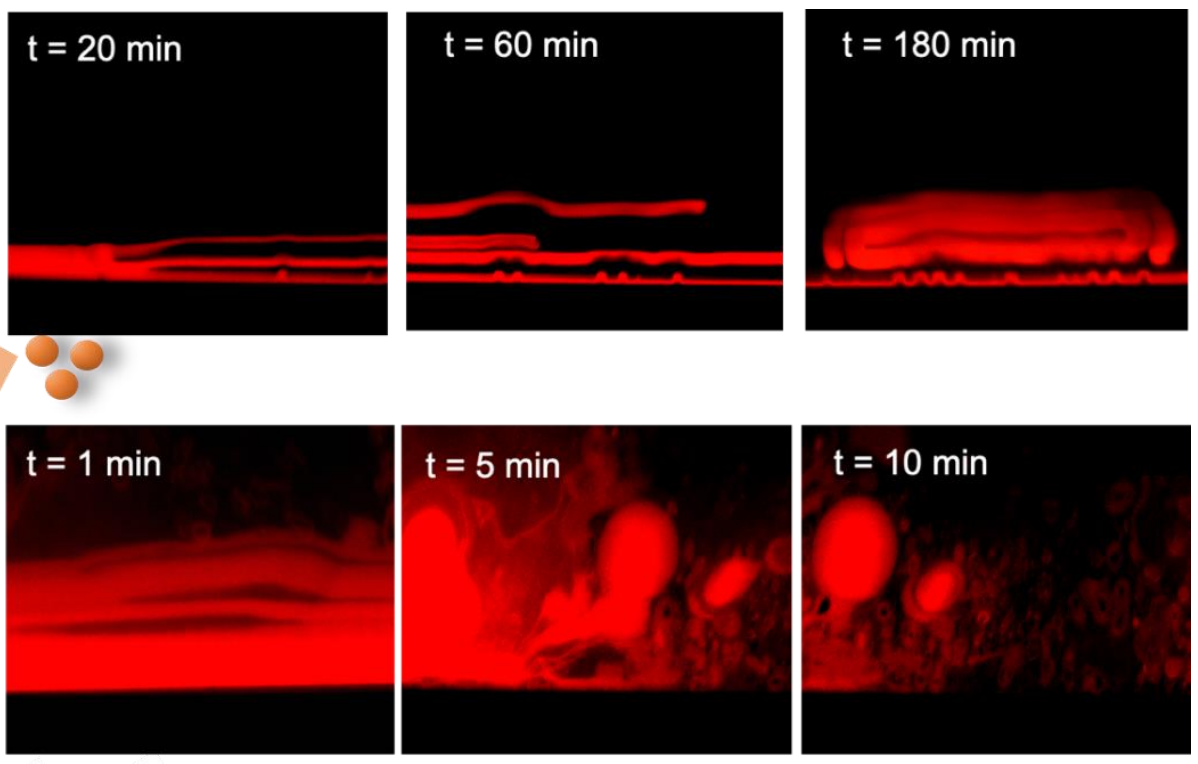

Figure 6 (a) CLSM images (lateral view) of AuNSs interacting with a GMO/DOPC lamellar film. From left to right: lamellar film before ( $t=0 \mathrm{sec}$ ), after $20 \mathrm{~min}, 60 \mathrm{~min}, 180 \mathrm{~min}$ from the addition of AuNSs. (b) CLSM images (lateral view) of AuNRs. Interacting with a GMO/DOPC lamellar film. From left to right: lamellar film before ( $\mathrm{t}=0 \mathrm{~min}$ ), after $1 \mathrm{~min}, 5 \mathrm{~min}, 10 \mathrm{~min}$ from the addition of AuNRs

This process leads to the progressive peeling-off of the lamellar film (60 min), with a gradual detachment of the outer surface layers. Once removed from the original matrix, these lipid layers start to bend and fold, ultimately rolling up in closed onion-like vesicular structures (180 min), which partially attach to the lipid film surface. After $180 \mathrm{~min}$ of incubation, only a thin layer of the original lamellar film is preserved onto the glass surface, partially covered by micron-sized multilamellar vesicles. The addition of asymmetrically shaped AuNRs (Figure 6b) has a similar impact on the film morphology, which seems to progress in a similar way as with the AuNSs. However, the overall process is faster, consisting of an initial massive swelling of the lamellar membrane, which is observed after just $1 \mathrm{~min}$ from the AuNRs addition. Moreover, the peelingoff of the film starts already after $5 \mathrm{~min}$ and, differently from the case of AuNSs, leads to the complete disruption of the lamellar film within $10 \mathrm{~min}$, with only polydisperse vesicular structures remaining adsorbed onto the glass surface.

A completely different behaviour is observed when cubic phase lipid films are exposed to the same AuNPs. Spherical NPs added at the same concentration (Figure 7a) seem to "excavate" the cubic membrane, producing an initial thinning of the film over selected areas (see the highlighted area in Figure 6a, 30 min). 

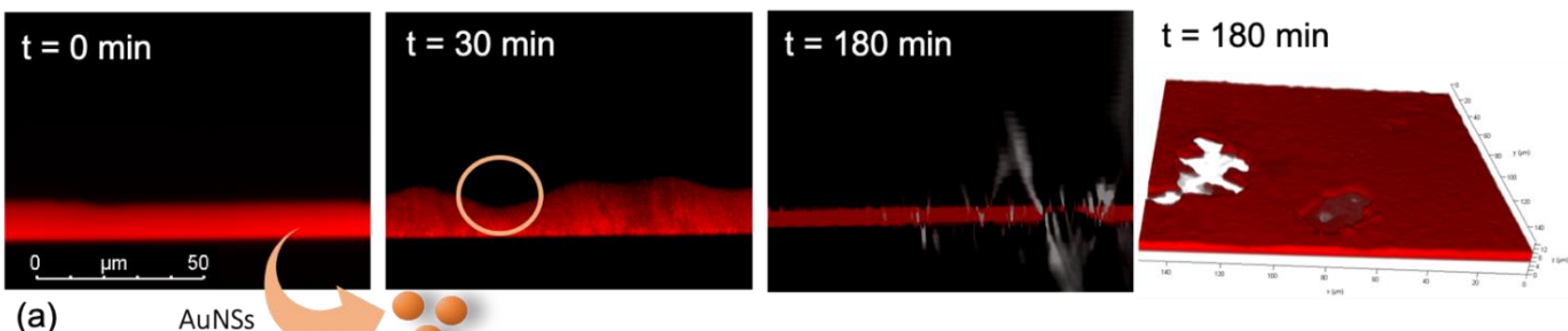

(a) AuNSs
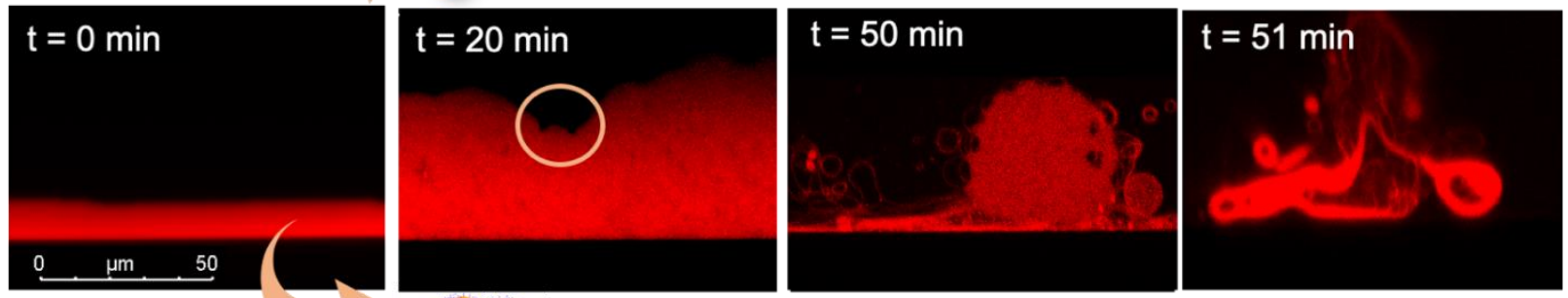

(b)

AuNRs

Figure 7 (a) CLSM images (lateral view) of AuNSs interacting with a GMO cubic film. From left to right: lamellar film before ( $\mathrm{t}=0 \mathrm{~min}$ ) and after $30 \mathrm{~min}, 180 \mathrm{~min}$ from the addition of NSs. A 3D reconstruction of the film after 180 min interaction is also shown, highlighting the presence of micron sized holes in the film. (b) CLSM images (lateral view) of AuNRs interacting with a GMO cubic film. From left to right: cubic film before ( $\mathrm{t}=0 \mathrm{~min}$ ) and after $20 \mathrm{~min}, 50 \mathrm{~min}, 51 \mathrm{~min}$ from the addition of AuNRs.

The progressive excavation leads to the formation of cavities, whose depth increases with time, eventually reaching the glass surface. After 180min of incubation, the lipid film, although mostly intact, presents micron-sized holes, which are clearly highlighted from the 3D reconstruction of the film, reported in Figure 7a. As previously observed for the case of lamellar membranes, the addition of AuNRs produces faster and more profound morphological modifications (Figure 7b): AuNRs initially increase the films roughness (20 min), with the formation of "hills and hollows" across the micrometric membrane. Similarly to what was previously observed for spherical AuNSs, the lipid film is progressively excavated, showing micron-sized cavities, whose depth increases with time. However, in this case the erosion process is faster and progresses within a few minutes. Complete retraction of the cubic film occurs in localized areas on the glass substrate, giving rise to large spheres of lipid matrix (50 min). After $50 \mathrm{~min}$, the lipid film is completely dewetted, leaving only isolated lipid spheres onto the glass substrate. Then, these lipid spheres are decomposed/removed due to the presence of the AuNRs (51 min). 
The disruption process, occurring within few seconds, can be analysed in detail, by following the temporal evolution of a horizontal section of the droplet. For this purpose, top-view images of a lipid droplet from formation ( $0 \mathrm{sec}$ ) and after different have been acquired in fluorescence and transmission mode. This allowed us to simultaneously capture the fluorescence of the Nile Red labelled lipid matrix, and gold nanoparticles. Representative images acquired within 10 sec from the droplet formation are reported in Figure 8a.

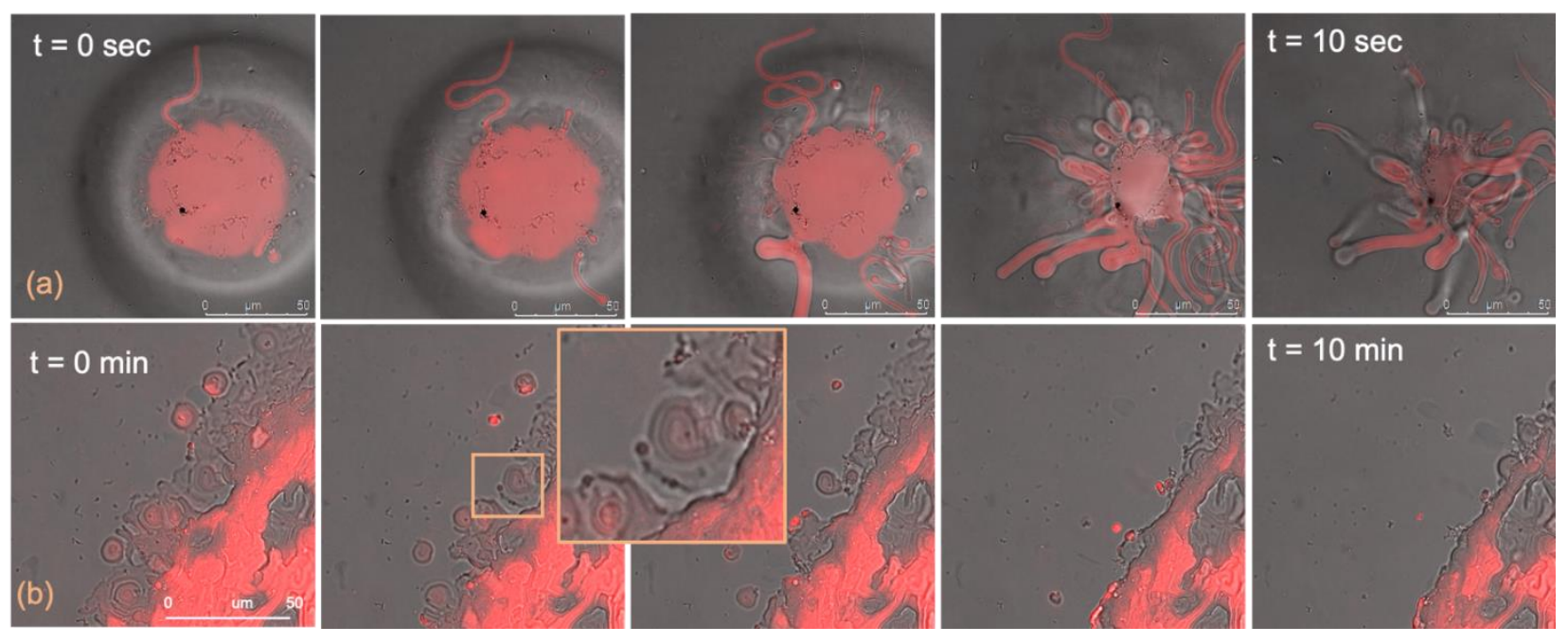

Figure 8 CLSM images of AuNRs interacting with a GMO cubic film (overlay of fluorescence and transmission images): (a) Disruption mechanism of a cubic film dewetted droplet by AuNRs, through the sequential extraction of lipid tubules from the bulk. (b) Mechanism of film excavation by AuNRs.

At the beginning of the process, AuNRs, which can be visualized as black spots in the image, are located as micron-sized clusters at the edges of the lipid droplet. Then, they start to excavate and unroll the lipid droplet starting from edges of the sphere. This led to complete collapse of the sphere within $10 \mathrm{sec}$ (see also the SI video). In addition, it is possible to further analyse the mechanism of interaction of AuNRs with the lipid films by monitoring their excavation of the film in detail (Figure 8b). Briefly, the AuNRs concentrate at the edges of the film as clusters, which progressively interact with the lipid interface and erode it. Interestingly, these clusters (which are highlighted with a zoom in Figure 8b), appear to be elongated, which probably are formed by endto-end interactions of the AuNRs. On the contrary, for AuNSs only small clusters of round shape appear on the lipid film (see SI figure S3).

Overall, the results gathered lead to two main conclusions: i) in agreement with the NR analysis, the impact of AuNRs on both lamellar and cubic model films is stronger and leads to faster structural and morphological modifications with respect to AuNSs; ii) NPs interact with lamellar and cubic phases according to two different and well-defined mechanisms, i.e. the peeling-off of the lamellar assembly and the excavation-dewetting of the cubic phase assembly. 


\section{The role of symmetry in NPs-lipid films interaction}

The experimental results obtained with the different experimental techniques combined give a clear picture of the role that structure and morphological plays to control NPs interactions with model membrane at different length scales. The mechanism is strongly affected by the structure of both the lipid film, e.g. the phase behaviour, and morphology, i.e. shape asymmetry of the NPs, as briefly summarized in Figure 9 .

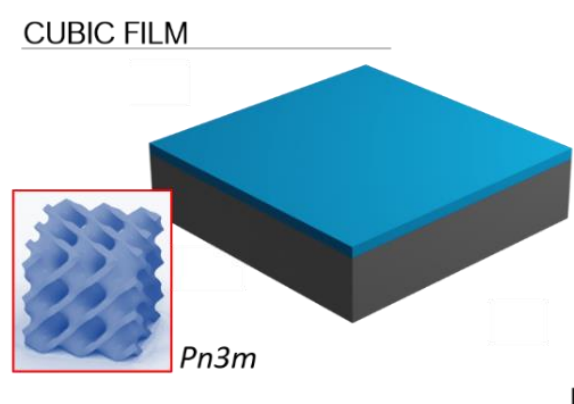

\section{LAMELLAR FILM}
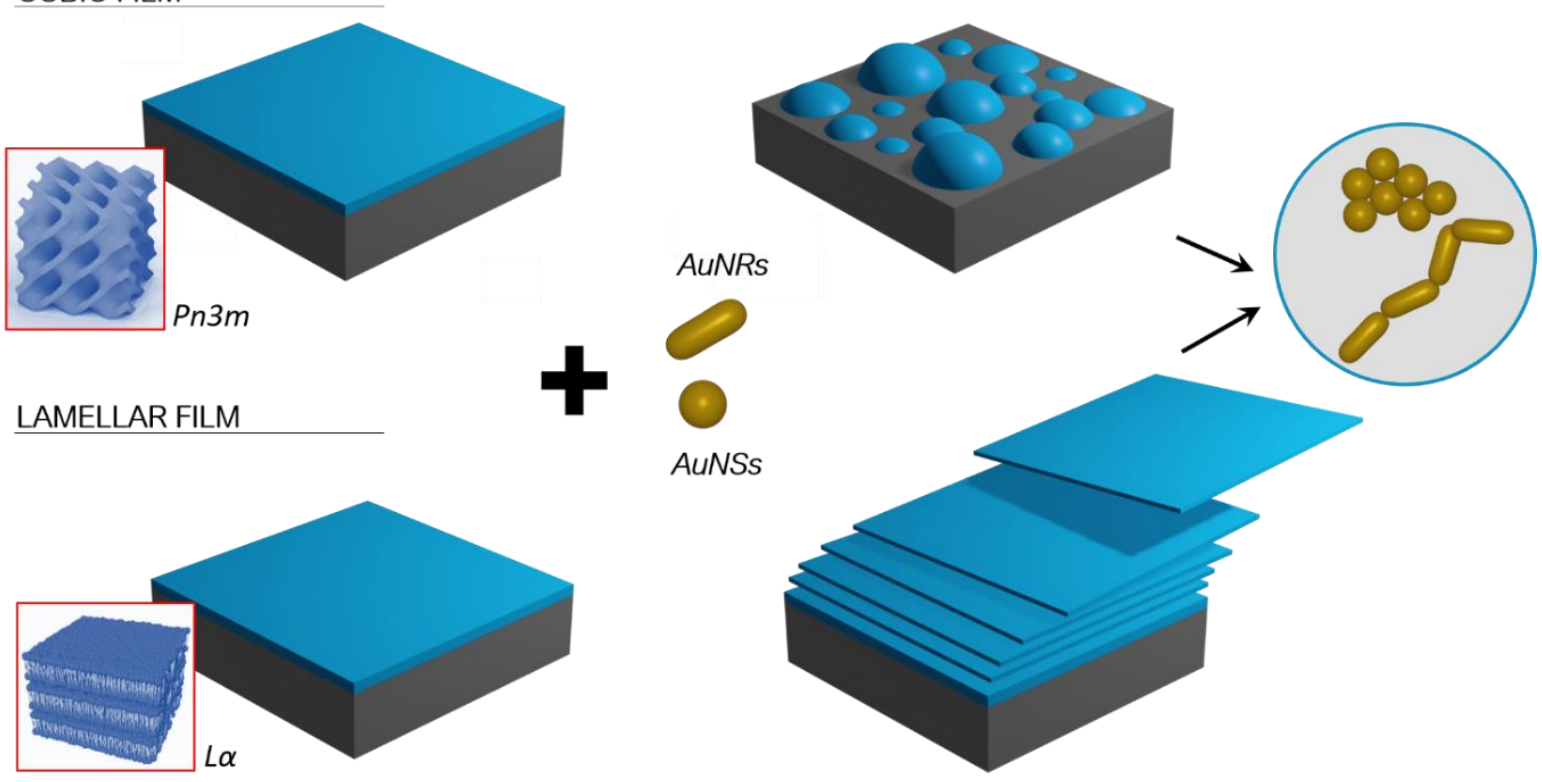

Figure 9 Schematic illustration of the results of the interaction between AuNPs and cubic and lamellar phase lipid films. AuNPs induce the excavation and then dewetting of the cubic films (on the top), while provoking a progressive exfoliation of the lamellar arrangement (on the bottom). The disruption of both cubic and lamellar films is faster with AuNRs than with AuNSs, related to the different clustering processes of AuNPs interacting with lipid membranes, i.e. symmetrical clustering of AuNSs versus edge-to-tail asymmetric aggregation of AuNRs).

\section{The effect of NPs shape asymmetry}

Both NR and CLSM results point out a major role of NPs asymmetric shape in the interaction with model membranes. NR results show that AuNRs have a profound impact on the structure of both lamellar and cubic films, where a complete loss of structural order occurs in less than 15 min and after $2 \mathrm{~h}$ for L $\alpha$ and Pn3m phases, respectively. On the contrary, the combination of NR and GISANS analyses shows significantly less effect of the AuNSs on the lipid films. Indeed, we only detected a partial disruption of lamellar and cubic structures after $13 \mathrm{~h}$ and $32 \mathrm{~h}$ of incubation, respectively. These observations are consistent with CLSM findings, highlighting the direct link 
between the changes of morphology on micron-scale and of structure on the nanoscale induced by the NPs.

Our results support numerous findings in literature, according to which the impact of NPs surface functionalization, composition and size on lipid membranes are recognized as the key determinants in NPs cytotoxicity ${ }^{1,5,29,41-45}$. However, much less is known on the effect of the NP shape on both model and natural biomembranes, and a some of the reported observations are contradictory. Asymmetric NPs have higher surface area/volume ratio, which is theoretically predicted to maximize the surface available to interact and attach to membranes. The main driving force here are Van der Waals and possible electrostatic attractive forces ${ }^{1,16}$, as well as hydrophobic interaction, depending on the NP properties. This has been often connected to a high affinity to bilayer model ${ }^{29}$ and natural membranes ${ }^{46}$, which in turn has been connected to an enhanced cells uptake ${ }^{47,48}$. On the other hand, the higher surface curvature at the edges of nonspherical NPs is predicted to increase the energy barrier for membranes wrapping the NPs that can drive the internalization by cells ${ }^{3}$. Recent experimental findings report lower cellular uptake of asymmetric NPs compared to spherical ones ${ }^{14,15}$. In addition, to the best of our knowledge, the impact of NP shape on their interaction with nonlamellar lipid films, mimicking curved bilayers, has to our knowledge not been studied before.

Here, employing spherical and rod-like AuNPs of comparable diameter and surface charge, we can separately study the effect of NPs shape on their interaction with membranes. We found that, when NPs size is similar to the lipid bilayer thickness, shape is of major importance, with asymmetric geometries producing more destructive effects on the lipid film structure and integrity. In particular, CLSM results suggest that, thanks to their asymmetric nature, AuNRs are able to maximize their interfacial interaction with the lipid films, by forming end-to-end elongated clusters while AuNSs seem to form more spherical clusters. Our results suggest that formation of the NP elongated clusters facilitate extraction lipid tubules from the film. Eventually this was found leading to the complete erosion and disruption of the layer (see Figure 9 scheme). Importantly, this behavior was observed for both lamellar and non-lamellar films, highlighting a universal effect of NPs asymmetry on membranes of different structures. 


\section{The effect of membrane phase structure}

Besides NPs shape, we found that membrane phase structure was another key factor that controls NPs interactions. NR and CLSM analyses highlighted that, for a given NP type, membrane geometry determines both the strength, pathway and results of interaction with NPs. Specifically, our NR results show that, independently from their shape, NPs induce a faster disruption of the nanostructure of lamellar films compared to cubic ones. This results into more rapid morphological modifications at the micron-scale as observed using CSLM. By combining the NR and CSLM data, we defined two different mechanisms that describe the interaction of NPs with lamellar and cubic phases and are responsible for their different stability towards nanomaterials (see Figure 9).

The faster structural modification of La films induced by both AuNSs and AuNRs (NR and GISANS analyses) is associated with an initial swelling, followed by progressive exfoliation of the multilamellar arrangement (CSLM analysis), proceeding from the outer lamellar layers. We can hypothesize that, after their injection, NPs start to penetrate the outer part of the lamellar arrangement, i.e. the part directly exposed to water. This penetration would primarily occur thanks to electrostatic and dipolar interactions between the cationic coating of NPs and the polar headgroup of lipids composing the membrane. This interaction would enable the insertion of NPs, together with their hydration shell, within the hydrophilic domains of the phase, i.e. the water layers separating the different lamellae. Then, the mismatch between the thickness of the water layers (around 2-3 nm) and NPs hydrodynamic size (see Dynamic Light Scattering results in the SI) would be responsible for the initial swelling of the lamellar film, observed through CSLM. The increasing inter-lamellar distance would reduce the interactions between different lamellae, producing the detachment of the outer lamellar layers, which are the first ones to be in contact with NPs. Indeed, lipid bilayers of equal composition are held together only by weak Van Der Waals forces, in equilibrium with repulsive electrostatic and entropic contributions ${ }^{49}$. Thus, interbilayer interactions are easily overcome by attractive single bilayer-NPs forces of electrostatic nature. The thinning of the film favors the penetration of NPs deeper into the film, i.e. coming closer to the solid support and promoting progressive peeling-off the lipid film. The loss of the lamellar periodicity as the layers are being detached can be connected with the progressive smearing out of the La-structural features, observed through NR (Figures 2, 4, 5).

On the other hand, a different interaction mechanism can be expected for the case of cubic phase films, accounting for their higher structural stability. NR and GISANS data highlight a progressive disordering and disruption of the cubic Pn3m nanostructure induced by NPs, which starts from 
the shrinkage of its lattice parameter (see GISANS analysis). This nanoscale phenomenon coincides with a progressive excavation of the film observed at the micron-scale (CSLM analysis), leading to de-wetting. Interestingly, this phenomenon proceeds from NPs clusters, which, acting like micron-sized "diggers", progressively erode and unroll the lipid matrix. We can hypothesize that, in this case, NPs are not able to fully penetrate the internal aqueous region of the film. Indeed, cubic phases represent a 3D highly interconnected network, where a single lipid bilayer folds in the space originating bicontinuous lipid and aqueous domains ${ }^{20}$, where the aqueous nanochannels has a diameter of around $4.4 \mathrm{~nm}$. This liquid crystallin 3D architecture would impede a full insertion of NPs within the smaller aqueous channels. Instead, the attractive NPslipid headgroups forces would result in the absorption of NPs, residing onto the film surface as clusters. As highlighted by NR analysis, their presence induce an immediate shrinking of the phase, leading to local release of water and dehydration of the phase. We can speculate that this phenomenon drives the progressive collapse of the cubic arrangement. Such process would then proceed from the different points where NPs accumulation across the membrane. Ultimately this leads to isolated of lipid droplets onto the solid substrate.

The enhanced stability of cubic phases compared to lamellar ones might have important biological implications. As already discussed, curved membranes in assemblies with cubic symmetry are known to form in cells under certain conditions (e.g., viral infection, oxidative stress, starving, membrane fusion and formation of extracellular vesicles) However, cubic membrane-related investigations has been limited at a descriptive level, while the biological function of these arrangements remains unexplored ${ }^{20}$. In particular under pathological conditions, it is still unknown whether their formation is solely a result of aberrant processes within diseased cells or represent a functional response to these pathologies.

Engineered NPs often have similar size range as biologically relevant entities (i.e., DNA, surface proteins, biogenic extracellular vesicles, and viruses), often resulting in similar interaction pathways with cells ${ }^{1}$. Thus, we might connect the higher resilience exhibited by cubic membranes towards NPs with a similar behavior towards natural nano-objects, such as viral pathogens. Within this perspective, our results seem to suggest a possible "protective role" of the cubic architecture, occurring in critical cell conditions to minimize the membrane response towards harmful external perturbations.

To summarize, we found that NPs-lipid membrane interactions depend both on the nanostructure of the membrane and the shape of NPs. These two variables represent two sides of the same coin, as a single parameter, i.e. symmetry, can describe their variations. In these terms, we can 
state that NPs with asymmetric shape promote a stronger interaction with the lipid matrix. On the other hand, when referring to the structure and morphology of the lipid membrane, we observed that flat 2D membranes experience more substantial disruptive effects than curved ones with 3D symmetry when exposed to NPs. Importantly, we also pointed out that a variation in NPs symmetry only affects the strength of interaction with lipid films, but not the interaction pathway; on the contrary, membranes with different morphologies lead to completely different interaction mechanisms. This is the basis for the observed differences in the structural resilience of these systems.

\section{Experimental}

\section{Materials}

Tetrachloroauric (III) acid ( $\geq 99.9 \%$ ), (11-Mercaptoundecyl)-N,N,N-trimethylammonium bromide $(\geq 90 \%)$, Cetyl trimethyl ammonium bromide (99\%), octanethiol (98.5\%), ascorbic acid (98\%), AgNO3 (99\%), $\mathrm{HCl}$, toluene (99.8\%), $\mathrm{MeOH}$ (99.8\%), $\mathrm{EtOH}$ (99.8\%), $\mathrm{CHCl} 3$ (99\%), Tetraoctylammonium bromide (98\%), NaBH4 (98\%), n-hexane (99\%), 1,2-dioleoyl-sn-glycero-3phosphocholine (DOPC) and Nile Red ( $\geq 98 \%$ ) were provided by Sigma-Aldrich (St. Louis, MO). A glycerol monooleate sample (mono- and diglycerides ratio $44: 1$ by weight), denoted as RYLO ${ }^{\mathrm{TM}}$ MG19 Glycerol Monooleate (GMO), was produced and provided by Danisco Ingredients (now Dupont, Brabrand, Denmark) with the following fatty acid composition (Lot No. 2119/65-1): 89.3 $\%$ oleic, $4.6 \%$ linoleic, $3.4 \%$ stearic and $2.7 \%$ palmitic acid. All chemicals were used as received. Milli-Q grade water was used in all preparations.

\section{Synthesis of Gold Nanospheres (AuNSs)}

Gold nanospheres functionalized with the cationic derivative N,N,N-trimethyl(11mercaptoundecyl)ammonium bromide (TMA) were synthesized as described by Mclntosh et al. 27,28. First, octanethiol-capped spherical gold nanoparticles (NSs) were prepared following the two-phase method developed by Brust et al. ${ }^{25,26}$ : an aqueous solution of $\mathrm{HAuCl}_{4}(15 \mathrm{~mL}, 30 \mathrm{mM})$ was mixed with a solution of tetraoctylammonium bromide (TOAB) in toluene $(40 \mathrm{~mL}, 50 \mathrm{mM})$. The two-phase mixture was vigorously stirred until all the $\mathrm{HAuCl}_{4}$ was transferred from the aqueous solution into the organic phase; TOAB acts as the phase-transfer reagent. Octanethiol $(7.81 \mu \mathrm{L})$ was then added to the organic phase. A freshly prepared aqueous solution of sodium 
borohydride (12.5 mL, 0.4 M) was slowly added with vigorous stirring. On addition of the reducing agent, the organic phase changed colour from orange to deep brown within a few seconds. After further stirring for 3 hours, the organic phase was separated, evaporated to $5 \mathrm{~mL}$ in a rotary evaporator and mixed with $200 \mathrm{~mL}$ ethanol to remove excess thiol. The mixture was kept for 4 hours at $-18^{\circ} \mathrm{C}$ until a dark brown precipitate was formed, and the supernatant was removed with a pipette; the precipitate was washed with $200 \mathrm{~mL}$ of ethanol and put again in the freezer. After 4 hours, the ethanol supernatant was removed with a pipette and completely evaporated in a rotary evaporator, obtaining octanethiol-capped gold nanoparticles. NSs capped with TMA were prepared by stirring $100 \mathrm{mg}$ of octanethiol-capped NSs and $150 \mathrm{mg}$ of N,N,N-trimethyl(11mercaptoundecyl)ammonium bromide in $20 \mathrm{~mL}$ of degassed tetrahydrofuran under argon for two days at room temperature. The black precipitate of the gold nanoparticles was purified by repeated suspension, centrifugation, and decantation with dichloromethane. NSs capped with TMA were then dissolved in pure water without the need for $\mathrm{pH}$ adjustment.

\section{Synthesis of Gold Nanorods (AuNRs)}

Gold NRs were synthesized according to a newly developed seedless growth protocol by ElSeyed ${ }^{50}$. Briefly, $\mathrm{HAuCl}_{4}(5.0 \mathrm{~mL} ; 1.0 \mathrm{mM})$ was added to $5.0 \mathrm{~mL}$ of Cetyl trimethyl ammonium bromide (CTAB, $0.2 \mathrm{M})$ at $27^{\circ} \mathrm{C}$, under magnetic stirring. Then, $\mathrm{AgNO}_{3}(250 \mu \mathrm{L} ; 4.0 \mathrm{mM})$ was added. Subsequently, $\mathrm{HCl}(8.0 \mu \mathrm{L}, 37 \%)$ was added to obtain a pH of $1-1.15$. Then, we added 70 $\mu \mathrm{L}$ ascorbic acid $(78.8 \mathrm{mM})$ under magnetic stirring and waited until the solution was clear. Immediately afterward, Ice-cold $\mathrm{NaBH}_{4}(15 \mu \mathrm{L} ; 0.01 \mathrm{M})$ was added and allowed to react overnight. The final dispersion of AuNRs capped with CTAB was characterized by a dark pink colour. The excess of CITAB was removed from the dispersion by 10 cycles of centrifugation, each followed by precipitation of CITAB crystals and removal of the supernatant containing AuNRs.

\section{Preparation of lamellar and cubic lipid films}

We obtained lamellar and cubic phase lipid films from GMO/DOPC 50/50 $\% \mathrm{~mol} / \mathrm{mol}$ and pure GMO, respectively. The lipid solutions in $n$-hexane (30/70 lipid/hexane $\% \mathrm{w} / \mathrm{w})$ were spin-coated onto a solid substrate for $10 \mathrm{~s}$ at 700 r.p.m and then for $60 \mathrm{~s}$ at 2000 r.p.m. Most of the solvent evaporates during this procedure. The lipid-coated substrate was then immediately immersed in excess Milli Q water, leading to lamellar or cubic lipid films by hydration. For the case of lipid films 
prepared for CLSM analysis, lipid formulations were labelled with the hydrophobic dye Nile Red ( $0.1 \mathrm{~mol} \%$ with respect to the total lipid amount), enabling the visualization of lipid layers; $100 \mu \mathrm{L}$ of the lipid solution in $\mathrm{n}$-hexane were deposited onto a hydrophilic round glass substrate (diameter of $15 \mathrm{~mm}$ ) prior to spin-coating. Lipid films formed onto the glass substrate were then sealed into a single-well sample holder and hydrated with $2 \mathrm{~mL}$ of water before imaging. For the case of NR analysis, samples were formed by depositing $1 \mathrm{~mL}$ of $n$-hexane lipid solution on $50 \times 80 \times 15 \mathrm{~mm}^{3}$ ultra-polished Silicon n-doped single crystal (100) oriented (Siltronix, Archamps-France for the experiments performed at ISIS and Andrea Holm GmbH, Tann, Germany; roughness $\leq 5 \AA$ for the experiments performed at MLZ) to cover almost the whole surface. After spin-coating and hydration, the substrate was sealed into a flow-cell type sample holder. To remove any excess contamination and the remaining $n$-hexane, an excess of water (at least 25 times the sample cell volume, i.e. $1.8 \mathrm{~mL}$ ) was flushed through the sample cell. Silicon substrates were preliminary rinsed in either ultrapure water and ethanol, in order to remove organic residues. After that, they were bath sonicated for $30 \mathrm{~min}$ in ethanol with a Bandelin DL $1023 \mathrm{~L}$ bath sonicator, followed by other $30 \mathrm{~min}$ in ultrapure water (Millipore Simplicity UV). The surfaces were then cleaned with a Novascan PSD-UV8 UV/ozone plasma for $30 \mathrm{~min}$ and rinsed in ultrapure water. Finally, they were dried with nitrogen gas and stored in ultrapure water, ready for deposition.

\section{Neutron Reflectivity (NR)}

Static NR measurements (section 3.2) were carried out at the OFFSPEC reflectometer ${ }^{51}$ (Isis Neutron and Muon Source, Rutherford Appleton Laboratory, UK).

Neutrons in the wavelength range 1.0-14.5 $\AA$ were used to perform the measurements. Two incident angles, $0.50^{\circ}$ and $2.00^{\circ}$ allowed collecting data in the range $0.008 \leq \mathrm{Q} / \AA-1 \leq 0.3$. The arrival times and positions of scattered neutrons were detected on a $3 \mathrm{He} 1 \times 300 \mathrm{~mm}$ linear scintillator area detector (1.2 mm pixel size) positioned at $3.5 \mathrm{~m}$ from the sample. The linear detector also recorded the off-specular reflectivity in the vertical direction, which yields information about the in-plane structure at the interface. The detector consists of 768 wavelength shifting fibres with $0.5 \mathrm{~mm}$ pitch, resulting in an observable $Q x$ range of $-6.5 \times 10^{-4}$ to $6.5 \times 10^{-4} \AA^{-1}$. The observable Q-range corresponds to real space distances of approximately 1-40 $\mathrm{mm}$ (real and reciprocal space are inversely proportional and related via $d \approx 2 \pi / Q$ ). The set-up allows for a $\triangle Q z / Q z$ resolution of $2-5 \%$. In order to achieve a good signal-to-noise ratio, a counting time of about 5 hours for the measurement was used. 
NR kinetic measurements were performed at the REFSANS Horizontal TOF reflectometer operated by the Helmholtz-Zentrum Hereon at the Heinz Maier-Leibnitz Zentrum (MLZ) in Garching, Germany ${ }^{52}$. Neutrons in the wavelength range 3.0-21.0 $\AA$ were used to perform the measurements. A vertically collimated beam having a width of $40 \mathrm{~mm}$ was used to maximise the intensity. An incident angle of $3.0^{\circ}$ allowed collecting data in the range $0.032 \leq Q / \AA^{-1} \leq 0.22$ with a resolution of $\triangle Q z Q z=8 \%$, allowing covering the region in which the evolution of Bragg peaks as well as that of the off-specular scattering may be followed as a function of time. The arrival times and positions of scattered neutrons were detected on a Denex 2D $500 \times 700 \mathrm{~mm}^{2}$ multiwire

${ }^{3}$ He detector (pixel size $2.1 \times 2.9 \mathrm{~mm}^{2}$, efficiency $80 \%$ at $7 \AA$, gamma sensitivity $<10^{-6}$ ) positioned at $4.5 \mathrm{~m}$ from the sample. The detector was installed in a liftable vacuum tube forming an angle of $5.2^{\circ}$ with respect to the horizon. Reflectivities were acquired at time intervals of $5 \mathrm{~min}$, over a time of $5 \mathrm{~h}$. Raw data were reduced and converted to q-space images, obtaining Qx/Qz maps ${ }^{53}$. In all the evaluations, the vertical component $Q_{z}$ was corrected for the ballistic effect due to the gravitational field.

\section{Grazing Incidence Small Angle Neutron Scattering (GISANS)}

GISANS measurements were performed at the REFSANS, using most of the same settings adopted for carrying out NR measurements. The only differences concern the wavelength band composing the incident beam (ranging from 2.7 to $18.1 \AA$ with a wavelength resolution $\Delta M \lambda=5 \%$ ), which corresponds to different penetration depths within the sample. A radial collimator was used to focus the beam on the detector, placed at $10.2 \mathrm{~m}$ from the sample. Acquired data were divided in wavelength slices having a width of $10 \%$ with respect to the mean value. For a given wavelength slice, the two-dimensional intensity data sets describe different $(\mathrm{Qy}, \mathrm{Qz})$ ranges. As for the NR investigations, $z$ represents the direction which is normal to the liquid/nanoparticle interface; $x$ indicates the beam direction oriented towards the scattering beam, whereas $y$ is the remaining axis defining a levogyrous cartesian system. Also for the GISANS analysis, the Qz component was corrected for the ballistic effect.

GISANS was employed to investigate films' properties along the directions perpendicular and parallel to the substrate interface. The in-plane film structure can be studied from the scattering patterns on the $\left(Q_{y}, Q_{z}\right)$ plane. If $\alpha_{i}$ and $\alpha_{f}$ are the angles of incidence and reflection of the neutron beam, respectively, then the three spatial components of the scattering vector (which is defined 
as the difference between the wave vector of the scattered beam $\overrightarrow{\boldsymbol{k}_{\boldsymbol{f}}}$ and that of the incident beam $\left.\overrightarrow{\boldsymbol{k}_{\boldsymbol{\imath}}}\right)$ are:

$$
\left[\begin{array}{l}
Q_{x} \\
Q_{y} \\
Q_{z}
\end{array}\right]=\frac{2 \pi}{\lambda}\left[\begin{array}{c}
\cos \alpha_{f} \cos 2 \theta_{f}-\cos \alpha_{i} \\
\cos \alpha_{f} \sin 2 \theta_{f} \\
\sin \alpha_{i}+\sin \alpha_{f}
\end{array}\right]
$$

where $\lambda$ is the neutron wavelength denoted with, while $2 \theta_{f}$ indicates the scattering angle in the $x y$ plane, which is relevant to determine lateral correlation lengths. The angle of incidence ( $0.5 \mathrm{deg})$ was chosen to get slices above and below the wavelength-dependent critical angle for total reflection $(\alpha c)$. chosen was just above the critical angle for total reflection $\left(\alpha_{c}\right)$.

\section{Confocal Laser Scanning Microscopy}

A Leica CLSM TCS SP8 confocal microscope, operating in inverted mode, with a $63 \times 1.3$ numerical aperture water immersion objective, was used to image the lipid-based surface structures in excess water. The fluorescence of Nile Red lipophilic dye was excited at $561 \mathrm{~nm}$ and the fluorescence was acquired in the $571 \mathrm{~nm}-650 \mathrm{~nm}$ emission range, with a PMT. Images were taken with a resolution of $512 \times 512$ pixels using a $400 \mathrm{~Hz}$ bidirectional scan with each scanning line averaged four times. Leica software was used to create three-dimensional reconstructions of the z-stacks.

\section{Conclusions}

In this contribution we explored the impact of symmetry and shape anisotropy on nano-bio interactions, focusing both on the geometry of nanoparticles (NPs) and on the symmetry of the target membrane. By combining structural techniques with nanoscale resolution with Confocal Microscopy imaging, we connected structural and morphological modifications of lipid films induced by NPs, occurring at different length scales. Overall, our results show that breaking the 3D symmetry at the nano-bio interface (passing from a 3D sphere to a 1D rod in the NPs and from a 3D cubic phase to a 2D lamellar phase in the lipid film) results in an enhanced interaction. In addition, our findings provide new evidence on the role of cubic membranes in Nature, suggesting a protective function related to their 3D symmetry; in this perspective, cubic membranes would represent biological barriers with enhanced structural resilience, occurring in cells as a "last defence" under extreme conditions, as in infected, stressed, or starved cells.

\section{Conflicts of interest}


There are no conflicts to declare.

\section{Acknowledgements}

This work has been supported by the European Community through the evFOUNDRY project (H2020-FETopen, ID: 801367) and the BOW project (H2020-EIC-FETPROACT-2019, ID: 952183). The authors also acknowledge CSGI for the economic support. Dr. Mirko Severi is acknowledged for ICP-AES measurements; Heinz Maier-Leibnitz Zentrum (MLZ) (Garching bei München, Germany) and ISIS, Rutherford Appleton Laboratory Didcot, Oxfordshire (UK) are acknowledged for provision of beam-time.

\section{References}

1 M. Mendozza, L. Caselli, D. Berti and A. Salvatore, Nanoparticles and organized lipid assemblies: from interaction to design of hybrid soft devices inorganic stimuli responsive, Soft Matter, 2019, 15, 8951-8970.

2 R. Michel and M. Gradzielski, Experimental Aspects of Colloidal Interactions in Mixed systems of liposome and inorganic nanoparticle and their applications, Int. J. Mol. Sci., 2012, 13, 11610-11642.

3 S. Dasgupta, T. Auth and G. Gompper, Nano- and microparticles at fluid and biological interfaces, J. Phys. Condens. Matter, 2017, 29, aa7933.

4 C. M. Beddoes, C. P. Case and W. H. Briscoe, Understanding nanoparticle cellular entry: A physicochemical perspective, Adv. Colloid Interface Sci., 2015, 218, 48-68.

5 A. M. Farnoud and S. Nazemidashtarjandi, Emerging investigator series: interactions of engineered nanomaterials with the cell plasma membrane; what have we learned from membrane models?, Environ. Sci. Nano, 2019, 6, 13-40.

6 M. Henriksen-Lacey, S. Carregal-Romero and L. M. Liz-Marzán, Current challenges toward in vitro cellular validation of inorganic nanoparticles, Bioconjug. Chem., 2017, 28, 212221.

7 E. Blanco, H. Shen and M. Ferrari, Principles of nanoparticle design for overcoming biological barriers to drug delivery, Nat. Biotechnol., 2015, 33, 941-951. 
8 P. Falagan-Lotsch, E. M. Grzincic and C. J. Murphy, One low-dose exposure of gold nanoparticles induces long-term changes in human cells, Proc. Natl. Acad. Sci. U. S. A., 2016, 113, 13318-13323.

9 C. J. Murphy, A. M. Vartanian, F. M. Geiger, R. J. Hamers, J. Pedersen, Q. Cui, C. L. Haynes, E. E. Carlson, R. Hernandez, R. D. Klaper, G. Orr and Z. Rosenzweig, Biological responses to engineered nanomaterials: Needs for the next decade, ACS Cent. Sci., 2015, 1, 117-123.

10 L. Caselli, A. Ridolfi, J. Cardellini, L. Sharpnack, L. Paolini, M. Brucale, F. Valle, C. Montis, P. Bergese and D. Berti, A plasmon-based nanoruler to probe the mechanical properties of synthetic and biogenic nanosized lipid vesicles, Nanoscale Horizons, 2021, 543-550.

11 K. L. Chen and G. D. Bothun, Nanoparticles Meet Cell Membranes: Probing Nonspecific Interactions using Model Membranes, Environ. Sci. Technol., 2014, 48, 873-880.

12 A. H. Bahrami, Orientational changes and impaired internalization of ellipsoidal nanoparticles by vesicle membranes, Soft Matter, 2013, 9, 8642.

13 S. Dasgupta, T. Auth and G. Gompper, Wrapping of ellipsoidal nano-particles by fluid membranes, Soft Matter, 2013, 9, 5473-5482.

14 S. Dasgupta, T. Auth and G. Gompper, Shape and orientation matter for the cellular uptake of nonspherical particles, Nano Lett., 2014, 14, 687-693.

15 R. Vácha, F. J. Martinez-Veracoechea and D. Frenkel, Receptor-mediated endocytosis of nanoparticles of various shapes, Nano Lett., 2011, 11, 5391-5395.

16 Q. Mu, G. Jiang, L. Chen, H. Zhou, D. Fourches, A. Tropsha and B. Yan, Chemical Basis of Interactions Between Engineered Nanoparticles and Biological Systems, Chem. Rev., 2014, 114, 7740-7781.

17 E. Gonzalez Solveyra and I. Szleifer, What is the role of curvature on the properties of nanomaterials for biomedical applications?, Wiley Interdiscip Rev Nanomed Nanobiotechnol., 2016, 8, 334-354.

18 S. Nangia and R. Sureshkumar, Effects of nanoparticle charge and shape anisotropy on translocation through cell membranes, Langmuir, 2012, 28, 17666-17671. 
19 Z. A. Almsherqi, S. D. Kohlwein and Y. Deng, Cubic membranes: a legend beyond the Flatland * of cell membrane organization, 2006, 173, 839-844.

20 Z. A. Almsherqi, T. Landh and S. D. Kohlwein, Cubic Membranes : The Missing Dimension of Cell Membrane Organization, Elsevier Inc., 1st edn., 2009, vol. 274.

21 Y. Deng and M. Mieczkowski, Three-dimensional periodic cubic membrane structure in the mitochondria of amoebae Chaos carolinensis, 1998, 16-25.

22 D. P. Chang, J. Barauskas, A. P. Dabkowska, M. Wadsäter, F. Tiberg and T. Nylander, Non-lamellar lipid liquid crystalline structures at interfaces, Adv. Colloid Interface Sci., 2015, 222, $135-147$.

23 A. P. Dabkowska, M. Valldeperas, C. Hirst, C. Montis, G. K. Pálsson, M. Wang, S. Nöjd, L. Gentile, J. Barauskas, N. J. Steinke, G. E. Schroeder-Turk, S. George, M. W. A. Skoda and T. Nylander, Non-Lamellar lipid assembly at interfaces: Controlling layer structure by responsive nanogel particles, Interface Focus, , DOI:10.1098/rsfs.2016.0150.

24 A. Ridolfi, B. Humphreys, L. Caselli, C. Montis, T. Nylander, D. Berti, M. Brucale and F. Valle, Exploring the structure and mechanics of thin supported inverse bicontinuous cubic phase lipid films, bioRxiv, 2021, doi.org/10.1101/2021.03.29.437497.

25 S. Tatur and A. Badia, Influence of hydrophobic alkylated gold nanoparticles on the phase behavior of monolayers of DPPC and clinical lung surfactant, Langmuir, 2012, 28, 628-639.

26 M. Brust, M. Walker, D. Bethell, D. J. Schiffrin and R. Whyman, Synthesis of Thiolderivatised Gold Nanoparticles in a Two-phase Liquid-Liquid System, J. Chem. Soc., Chem. Commun., 1994, 801-802.

27 C. M. Mclntosh, E. A. Esposito, A. K. Boal, J. M. Simard, C. T. Martin and V. M. Rotello, Inhibition of DNA transcription using cationic mixed monolayer protected gold clusters, J. Am. Chem. Soc., 2001, 123, 7626-7629.

28 S. Tatur, M. Maccarini, R. Barker, A. Nelson and G. Fragneto, Effect of functionalized gold nanoparticles on floating lipid bilayers, Langmuir, 2013, 29, 6606-6614.

29 C. Montis, V. Generini, G. Boccalini, P. Bergese, D. Bani and D. Berti, Model lipid bilayers mimic non-specific interactions of gold nanoparticles with macrophage plasma membranes, $J$. Colloid Interface Sci., 2018, 516, 284-294. 
30 D. E. Sands, Introduction to Cristallography, W.A. Benjamin, Inc., New York, NY., 1969.

31 V. Cherezov, J. Clogston, Y. Misquitta, W. Abdel-Gawad and M. Caffrey, Membrane protein crystallization in meso: Lipid type-tailoring of the cubic phase, Biophys. J., 2002, 83, 33933407.

32 G. Popescu, J. Barauskas, T. Nylander and F. Tiberg, Liquid crystalline phases and their dispersions in aqueous mixtures of glycerol monooleate and glyceryl monooleyl ether, Langmuir, 2007, 23, 496-503.

33 N. Kučerka, S. Tristram-Nagle and J. F. Nagle, Structure of fully hydrated fluid phase lipid bilayers with monounsaturated chains, J. Membr. Biol., 2006, 208, 193-202.

34 R. Negrini and R. Mezzenga, Diffusion, molecular separation, and drug delivery from lipid mesophases with tunable water channels, Langmuir, 2012, 28, 16455-16462.

35 P. Müller-Buschbaum, Grazing incidence small-angle neutron scattering: Challenges and possibilities, Polym. J., 2013, 45, 34-42.

36 S. J. Richardson, P. A. Staniec, G. E. Newby, N. J. Terrill, J. M. Elliott, A. M. Squires and W. T. Góźdź, Predicting the orientation of lipid cubic phase films, Langmuir, 2014, 30, 1351013515.

37 R. Kampmann, M. Haese-Seiller, V. Kudryashov, B. Nickel, C. Daniel, W. Fenzl, A. Schreyer, E. Sackmann and J. Rädler, Horizontal ToF-neutron reflectometer REFSANS at FRMII Munich/Germany: First tests and status, Phys. B Condens. Matter, 2006, 385-386, 1161-1163.

38 K. Yang and Y. Q. Ma, Computer simulation of the translocation of nanoparticles with different shapes across a lipid bilayer, Nat. Nanotechnol., 2010, 5, 579-583.

39 W. K. Fong, T. L. Hanley, B. Thierry, N. Kirby, L. J. Waddington and B. J. Boyd, Controlling the nanostructure of gold nanorod-lyotropic liquid-crystalline hybrid materials using near-infrared laser irradiation, Langmuir, 2012, 28, 14450-14460.

40 P. Vandoolaeghe, A. R. Rennie, R. A. Campbell, R. K. Thomas, F. Höök, G. Fragneto, F. Tiberg and T. Nylander, Adsorption of cubic liquid crystalline nanoparticles on model membranes, Soft Matter, 2008, 4, 2267-2277.

41 P. Vedantam, G. Huang and T. R. J. Tzeng, Engineered nanoparticles interacting with cells: size matters, J. Nanobiotechnology, 2013, 4, 13-20. 
42 C. Montis, L. Caselli, F. Valle, A. Zendrini, F. Carlà, R. Schweins, M. Maccarini, P. Bergese and D. Berti, Shedding light on membrane-templated clustering of gold nanoparticles, J. Colloid Interface Sci., 2020, 573, 204-214.

43 T. Pfeiffer, A. De Nicola, C. Montis, F. Carlà, N. F. A. Van Der Vegt, D. Berti and G. Milano, Nanoparticles at Biomimetic Interfaces: Combined Experimental and Simulation Study on Charged Gold Nanoparticles/Lipid Bilayer Interfaces, J. Phys. Chem. Lett., 2019, 10, 129-137.

44 B. Pelaz, G. Charron, C. Pfeiffer, Y. Zhao, J. M. De La Fuente, X. J. Liang, W. J. Parak and $P$. Del Pino, Interfacing engineered nanoparticles with biological systems: Anticipating adverse nano-bio interactions, Small, 2013, 9, 1573-1584.

45 A. Ridolfi, L. Caselli, C. Montis, G. Mangiapia, D. Berti, M. Brucale and F. Valle, Gold nanoparticles interacting with synthetic lipid rafts: an AFM investigation, J. Microsc., 2020, 280, 194-203.

46 S. Salatin, S. Maleki and A. Y. Khosroushahi, Effect of the surface modification, size, and shape on cellular uptake of nanoparticles, Cell Biol Int., 2015, 39, 881-890.

47 S. E. A. Gratton, P. A. Ropp, P. D. Pohlhaus, J. C. Luft, V. J. Madden, M. E. Napier and J. M. Desimone, The effect of particle design on cellular internalization pathways, PNAS, 2008, 105, 11613-11618.

48 R. Agarwal, V. Singh, P. Jurney, L. Shi, S. V Sreenivasan and K. Roy, Mammalian cells preferentially internalize hydrogel nanodiscs over nanorods and use shape-speci fi c uptake mechanisms, PNAS, 2013, 110, 1-6.

49 J. N. Israelachvili, Intermolecular and Surface Forces, 2011, vol. 53.

50 M. R. K. Ali, B. Snyder and M. A. El-Sayed, Synthesis and optical properties of small Au nanorods using a seedless growth technique, Langmuir, 2012, 28, 9807-9815.

51 R. M. Dalgliesh, S. Langridge, J. Plomp, V. O. De Haan and A. A. Van Well, Offspec, the ISIS spin-echo reflectometer, Phys. B Condens. Matter, 2011, 406, 2346-2349.

52 J.-F. Moulin and M. Haese, REFSANS: Reflectometer and evanescent wave small angle neutron spectrometer. Journal of large-scale research facilities, J. large-scale Res. Facil. JLSRF. 53 A. Hafner, P. Gutfreund, B. P. Toperverg, A. O. F. Jones, J. P. de Silva, A. Wildes, H. E. Fischer, M. Geoghegan and M. Sferrazza, Combined specular and off-specular reflectometry: 
elucidating the complex structure of soft buried interfaces, J. Appl. Crystallogr., 2021, 54, 924948. 


\section{Supplementary Information for:}

\section{Interaction of nanoparticles with lipid films: the role of symmetry}

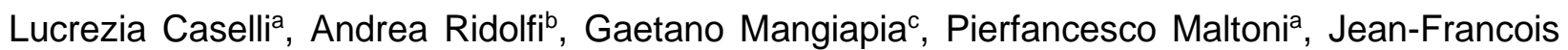
Moulin $^{c}$, Nina-Juliane Steinke ${ }^{d}$, Debora Berti, Emil Gustafsson ${ }^{e}$, Tommy Nylander ${ }^{f \star}$ and Costanza Montis $^{\text {a* }}$

${ }^{a}$ Department of Chemistry, University of Florence and CSGI, Florence (Italy); ${ }^{b}$ ISMN-CNR and CSGI, Bologna (Italy); ' Heinz Maier-Leibnitz Zentrum (MLZ), Garching bei München (Germany); 'ISIS, Rutherford Appleton Laboratory Didcot, Oxfordshire (UK); ' Department of Chemistry, Uppsala University, Uppsala (Sweden); 'Department of Chemistry, Lund University, Lund (Sweden).

\section{Small Angle X-ray Scattering}

SAXS measurements were carried out on a S3-MICRO SAXS/WAXS instrument (HECUS GmbH, Graz, Austria) which consists of a GeniX microfocus X-ray sealed Cu Ka source (Xenocs, Grenoble, France) of $50 \mathrm{~W}$ power which provides a detector focused X-ray beam with $\lambda=0.1542$ $\mathrm{nm} \mathrm{Cu} \mathrm{Ka} \mathrm{line.} \mathrm{The} \mathrm{instrument} \mathrm{is} \mathrm{equipped} \mathrm{with} \mathrm{two} \mathrm{one-dimensional} \mathrm{(1D)} \mathrm{position} \mathrm{sensitive}$ detectors (HECUS 1D-PSD-50 M system), each detector is $50 \mathrm{~mm}$ long (spatial resolution 54 $\mu \mathrm{m} /$ channel, 1024 channels) and cover the SAXS Q-range $\left(0.003<Q<0.6^{\circ} \AA-1\right)$. The temperature $\left(25^{\circ} \mathrm{C}\right)$ was controlled by means of a Peltier TCCS-3 Hecus. The analysis of SAXS curves was carried out using Igor Pro (Kline, 2006). SAXS measurements on AuNPs aqueous dispersions, were carried out in sealed glass capillaries of $1.5 \mathrm{~mm}$ diameter. The concentration of AuNSs in water was $1.1 \cdot 10^{15}$ particles $/ \mathrm{mL}$.

To analyze AuNSs scattering profile we chose a model function with a spherical form factor and a Schulz size distribution (Kotlarchyk and Chen, 1983) from the NIST package SANS Utilities. This model calculates the scattering for a polydisperse population of spheres with uniform scattering length density. The distribution of radii is a Schulz distribution given by the following equation:

$$
f(R)=(z+1)^{z+1} x^{z} \frac{\exp [-(z+1) x]}{R_{a v g} \Gamma(z+1)}
$$


where $R_{\text {avg }}$ is the mean radius, $x=R / R_{\text {avg, }} \mathrm{z}$ is a parameter related to the polydispersity and $\mathrm{G}(x)$ indicates the Gamma function. The form factor is normalized by the average particle volume, using the 3rd moment of $R$ :

$$
\langle V\rangle=\frac{4 \pi}{3}\left\langle R^{3}\right\rangle=\frac{4 \pi}{3}\langle R\rangle^{3} \frac{(z+3)(z+2)}{(z+1)^{2}}
$$

The scattering intensity is:

$$
I(Q)=\left(\frac{4 \pi}{3}\right)^{2} N_{0}(\Delta \rho)^{2} \int_{0}^{\infty} f(R) R^{6} F^{2}(Q R) d R
$$

where $N_{0}$ is the total number of particles per unit volume, $F(Q R)$ is the scattering amplitude for a sphere and $\Delta \rho$ is the difference in scattering length density between the particle and the solvent. The SAXS profile of AuNRs (at a concentration of $2.4 \cdot 10^{14}$ particles $/ \mathrm{mL}$ ) was fitted by the Cylinder poly radius model from the NIST package SANS Utilities; this model calculates the form factor for a polydisperse right circular cylinder with uniform scattering length density and a Schulz polydispersity of the cylinder length is considered. The function calculated is the orientationally averaged cylinder form factor which is then averaged over a Schulz distribution of the cylinder length $2 H$. The size averaged form factor is thus:

$$
P(Q)=\int_{0}^{\infty} \int_{0}^{\pi / 2} f(2 H) F^{2}(Q \alpha) \sin \alpha d \alpha d(2 H) d(2 H)
$$

where $\mathrm{f}(2 \mathrm{H})$ is the normalized Schulz distribution of the length $2 \mathrm{H}$. The limits of the integration are adjusted automatically to cover the full range of length. The scattering amplitude, F, is:

$$
F(Q \alpha)=2 V_{c y l}\left(\rho_{c y l}-\rho_{\text {solv }}\right) j_{0}(Q H \cos \alpha) \frac{J_{1}(Q R \sin \alpha)}{(Q R \sin \alpha)}
$$

Where $J_{1}(Q R \sin \alpha)$ is the first order Bessel function, $V_{c y l}=2 \pi H R^{2}, j_{0}(Q H \cos \alpha)=\frac{\sin (\mathrm{QH} \cos \alpha)}{\mathrm{QH} \cos \alpha}$, with $\alpha$ defined as the angle between the cylinder axis and the scattering vector $(Q)$ and $\rho_{\text {cyl }}$ and $\rho_{\text {solv }}$ the scattering length density of the nanorod and the solvent respectively. The integral over averages the form factor over all possible orientations of the cylinder with respect to $Q$. 


\section{Transmission Electron Microscopy (TEM)}

Trasmission electron microscopy (TEM) images were acquired with a STEM CM12 Philips electron microscope, at CeME (CNR Florence Research Area, Via Madonna del Piano, 10 50019 Sesto Fiorentino). The sample was placed on a 200 mesh carbon-coated copper grid.

\section{Dynamic Light Scattering (DLS)}

DLS measurements were performed using a Malvern Panalytical Zetasizer Nano ZS90 instrument which does DLS measurements at a fixed scattering angle of $90^{\circ}$. A $4 \mathrm{~mW}$ laser of $633 \mathrm{~nm}$ wavelength is used as light source, the lag times of the correlator start from $25 \mathrm{~ns}$ as shortest and go up to $8000 \mathrm{~s}$, using a maximum number of 4000 channels. After checking monomodality with a CONTIN fit, the ACFs were analyzed through the cumulant fitting stopped to the second order, allowing an estimate of the hydrodynamic diameter and the polydispersity of AuNSs and AuNRs, which were found equal to: $25.6 \pm 0.2$ and $37.6 \pm 0.1 \mathrm{~nm}$ (hydrodynamic diameter) with a 0.3 and 0.21 (PDI), respectively.

\section{Z-Potential}

Zeta potential measurements were performed using a Zeta Potential Analyzer (Zeta Plus, Brookhaven Instruments Corporation, Holtsville, NY). Zeta potentials were obtained from the electrophoretic mobility $u$, according to Helmholtz-Smoluchowski equation: $\zeta=(\eta / \varepsilon) \times u$ with $\eta$ being the viscosity of the medium, $\varepsilon$ the dielectric permittivity of the dispersing medium. The Zeta Potential values are reported as averages from ten measurements.

\section{Inductively Coupled Plasma Atomic Emission Spectroscopy (ICP-AES)}

This analysis was kindly done by Dr. Mirco Severi, in order to define the concentration of NPs dispersions, with an ICP-AES Varian 720-ES. For the analysis, $200 \mu \mathrm{L}$ of NPs dispersion were 
placed in a vial, then the solvent was evaporated under slight nitrogen flow. The sample, consisting of a dry film of nanoparticles, was diluted to $5 \mathrm{~mL}$ with $0.1 \%$ super pure nitric acid, obtained by distillation under boiling, then, to the sample, $1 \mathrm{ppm}$ of Ge was added, as internal standard; the sample thus treated was analysed.

The operating conditions for the ICP-AES analysis have been optimized to obtain the maximum signal intensity and are as follows:

Instrument: Varian 720-ES

Power R.F: $1.20 \mathrm{KW}$

Flow rate of Argon Plasma: $16.5 \mathrm{~L} \mathrm{~min}^{-1}$

Auxiliary Argon flow rate: $1.50 \mathrm{~L} \mathrm{~min}^{-1}$

Argon nebulizer flow rate: $0.75 \mathrm{~L} \mathrm{~min}^{-1}$

Replicated reading time: 5 seconds

Instrument stabilization time: 30 seconds

Sample introduction settings:

Sample uptake: 30 seconds

Flow rate: $1 \mathrm{~mL} \mathrm{~min}{ }^{-1}$

Rinse time: 70 seconds

Fast Pump (sample delay / rinse): active

Smart rinse: active

Replicates: 3

From the ICP-AES data, it results that the quantity of gold in $200 \mu \mathrm{L}$ of AuNSs and AuNRs dispersions is equal to $259 \mu \mathrm{g}$ and $278 \mu \mathrm{g}$; as a result, the concentrations of Au in AuNSs and AuNRs is $1.3 \mathrm{mg} / \mathrm{mL}$ and $1.4 \mathrm{mg} / \mathrm{mL}$, respectively. The AuNSs and AuNRs concentration per $\mathrm{ml}$ was subsequently evaluated considering the size of AuNPs determined through SAXS and is equal to $3.2 \cdot 10^{15}$ and $7.4 \cdot 10^{14}$ particles $/ \mathrm{mL}$ for AuNSs and AuNRs, respectively. 


\section{Evaluation of the spacing parameter of cubic and lamellar films through NR}

The unit cell spacing (d) of cubic and lamellar films was evaluated from NR data (i.e., with hkl Miller indices, hkl, (100) and (200)), using the Q-position of the Bragg reflection peaks of cubic and lamellar phases. In particular, the lamella phase features two prominent Bragg peaks located at 0.099 and $0.192 \AA^{-1}$, corresponding to the first two reflexes of the lamellar, $L_{\alpha}$, phase (i.e., with Miller indices, hkl, (100) and (200)). The unit cell spacing was calculated using the q-position of the maximum Bragg reflection peaks $d=2 \pi h / Q_{h}$, yielding a value of $6.4 \pm 0.1 \mathrm{~nm}$ as the mean between the two peak positions. The reflectivity profile of the GMO lipid film presents two clearly distinguishable Bragg peaks at q values 0.093 and $0.115 \AA^{-1}$, corresponding to Miller indices (110) and (111) of the Pn3m inverse cubic phase. The lattice spacing $\mathrm{d}$, can be calculated from $d=$ $2 \pi\left(h^{2}+k^{2}+l^{2}\right)^{1 / 2} / Q_{100}$ (Sands, 1969), yielding a value of $9.51 \pm 0.05 \mathrm{~nm}$ as the mean between the two peak positions.

\section{Evaluation of the spacing parameter of cubic films through GISANS}

In order to obtain the lattice parameter of the Pn3m cubic architecture (in the presence and in the absence of AuNSs), we used the GISANS images shown in the main text (Figure 3). We performed horizontal line cuts from the 2D GISANS data, along selected Qz values, obtaining the Intensity vs Qy plots, reported in Figure S2. The Qy values corresponding to the intensity's maxima were then used to determine the $Q$ value of a spot on the $Q_{y} / Q_{z}$ GISANS plot, according to following equation:

$$
Q^{2}=Q_{y}^{2}+Q_{z}^{2}
$$

The lattice parameter $(\mathrm{d})$ is then evaluated from the following equation: $d=2 \pi\left(h^{2}+k^{2}+l^{2}\right)^{1 / 2}$ / $Q_{h k l}$ (with $\mathrm{h}, \mathrm{k}$ and I miller indices identifying a Bragg reflection peak). $Q_{h k l}$ is taken as the mean between the Q-values obtained for the different intensity's spots of each horizontal line cut. 
a) GMO CUBICFILM
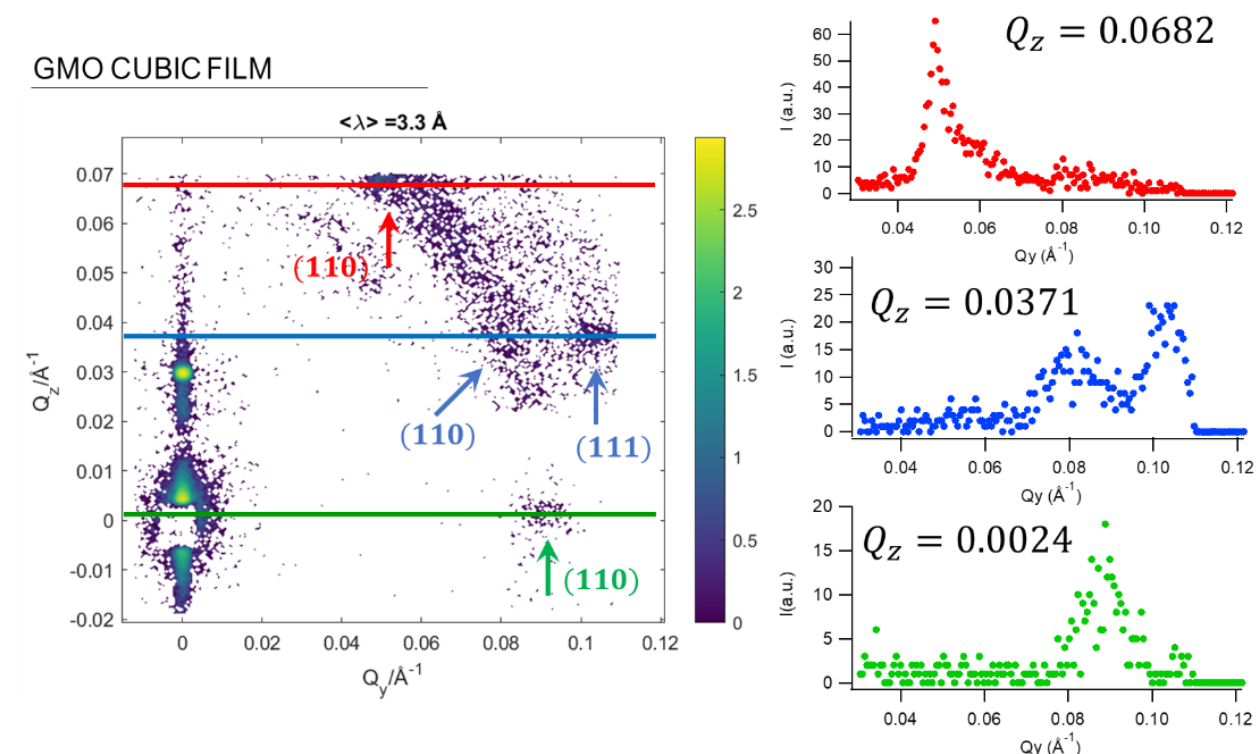

b)
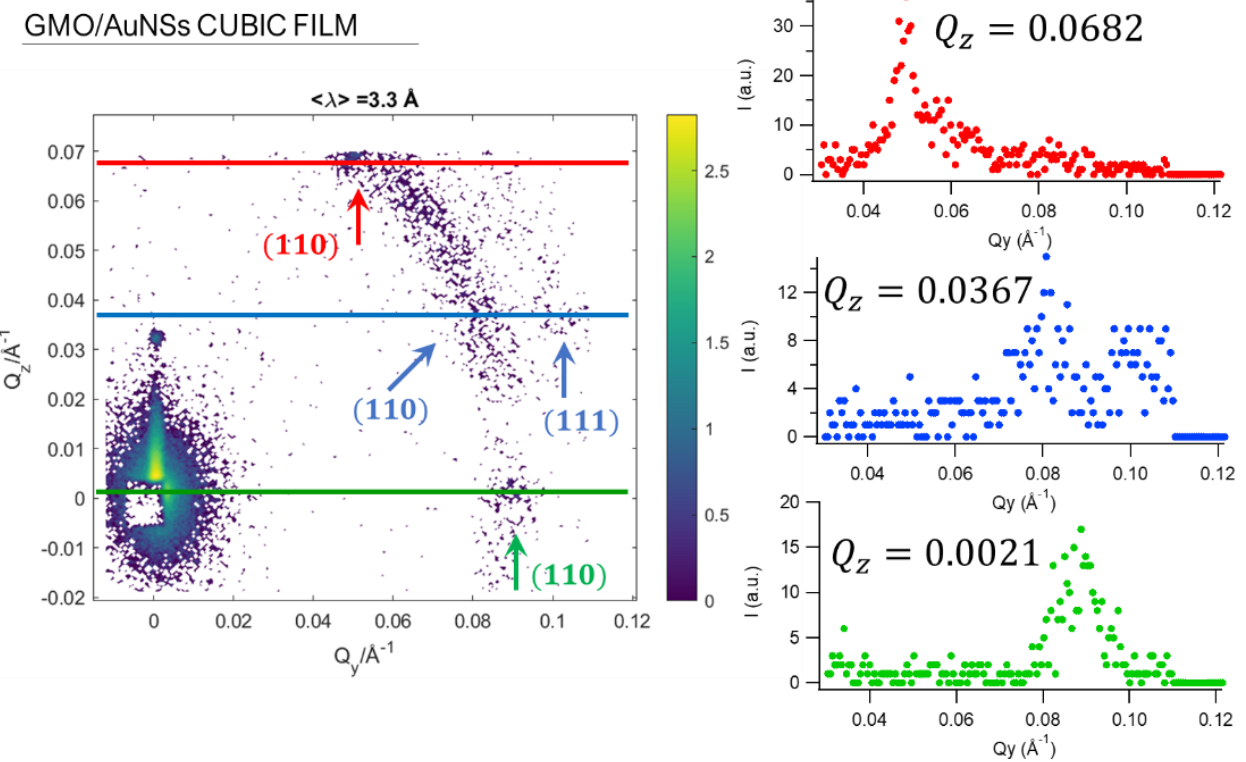

Figure S1: a) Left part: GISANS 2D plot of the GMO/water film; the horizontal lines represent the directions along which the line cuts are performed. Right part: horizontal Intensity vs Qy line cuts obtained from the 2D GISANS plot along different Qz. 


\section{Analysis of NR profiles of cubic and lamellar films interacting with AuNRs}
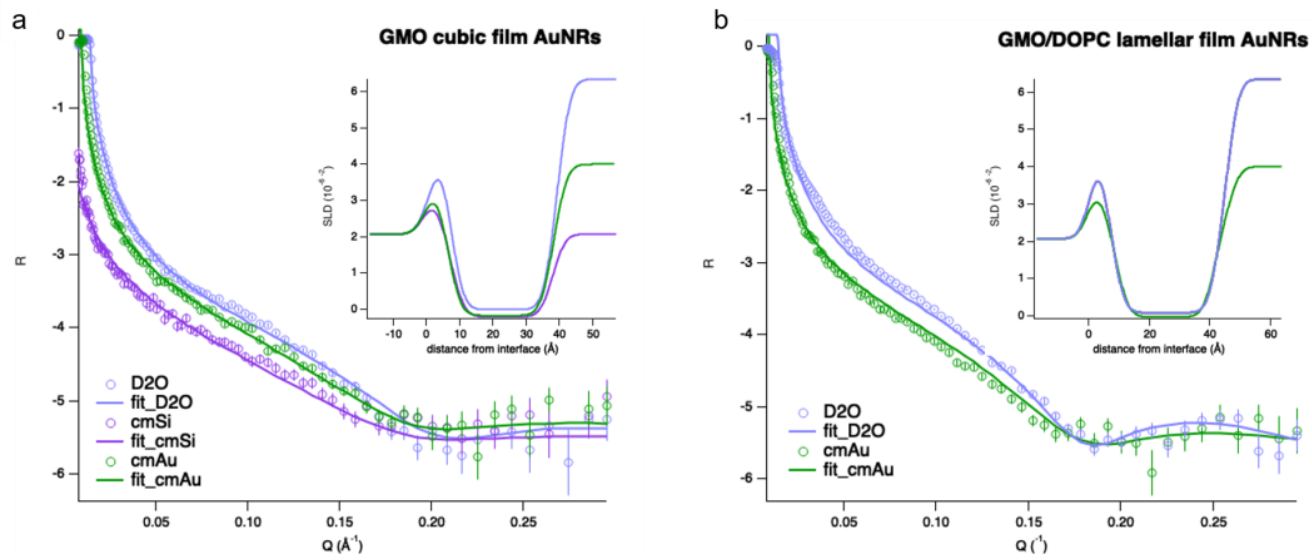

Figure S2: Neutron Reflectometry profiles of (a) GMO cubic films and (b) GMO/DOPC lamellar films upon interaction with AuNRs. Three contrasts were measured for GMO/AuNRs system: D2O (SLD 6.34e-6 $\AA^{-1}$ ), cmAu (contrast match gold, 4.6e-6 $\AA^{-1}$ ), cmSi (contrast match silicon, 2.07e-6 $\AA^{-1}$ ), while two contrasts were measured for GMO/AuNRs system (D2O, cmAu). It was possible to analyze both GMO (a) and GMO/DOPC (b) systems according to a five-layers model, accounting for the formation of a lipid bilayer. For GMO the bilayer thickness is $34+/-3 \mathrm{~nm}$, while for GMO/DOPC the bilayer thickness is $40+/-4 \mathrm{~nm}$, consistent with the parameters expected from the literature (ref). The bilayer thickness was obtained by analyzing the data with motofit (ref), considering scattering length density values of: $2.07 \mathrm{e}-6 \AA^{-1}$ (silicon layer), $3.41 \mathrm{e}-6 \AA^{-1}$ (Silicon oxide layer), 2.17e-6 $\AA^{-1}$ (GMO polar headgroup layer), -2.12e-7 $\AA^{-1}$ (GMO and DOPC lipid chain layer), 2e-6 $\AA^{-1}$ (mixed GMO/DOPC polar headgroup layer). In both cases, the layer appear homogeneous, with high coverage of the substrate (inferred from the low hydration degree of the systems $<10 \%$ )

\section{CLSM images of AuNSs clusters on lipid films}
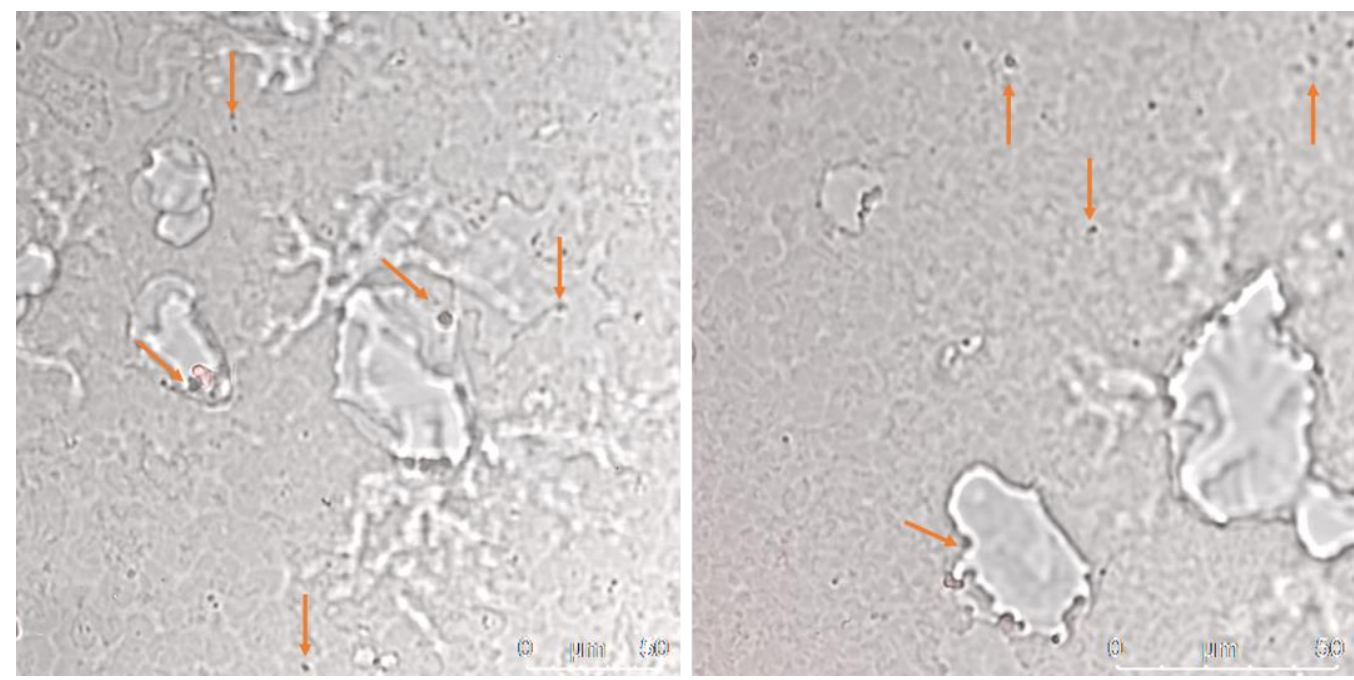
Figure S3: Representative transmission CLSM images of GMO cubic films incubated with AuNSs. The orange arrows in the figure highlight the presence of multiple round-shaped black spots attributable to the presence of AuNSs clusters.

\section{Bibliography}

Kline, S. R. (2006) 'Reduction and analysis of SANS and USANS data using IGOR Pro', Journal of Applied Crystallography, 39(6), pp. 895-900. doi: 10.1107/S0021889806035059.

Kotlarchyk, M. and Chen, S.-H. (1983) 'Analysis of small angle neutron scattering spectra from polydisperse interacting colloids', The Journal of Chemical Physics, 79(5), p. 2461. doi: 10.1063/1.446055.

Sands, D. E. (1969) Introduction to Cristallography. W.A. Benjamin, Inc., New York, NY. 